\title{
Changes to taxonomy and the International Code of Virus Classification and Nomenclature ratified by the International Committee on Taxonomy of Viruses (2018)
}

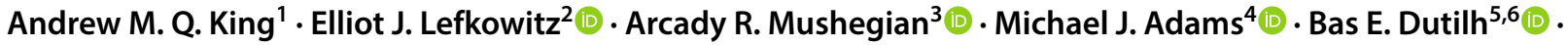

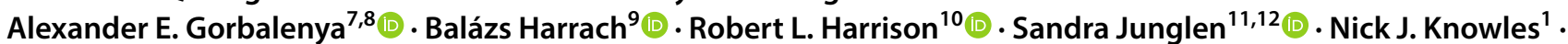

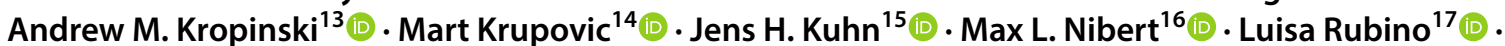

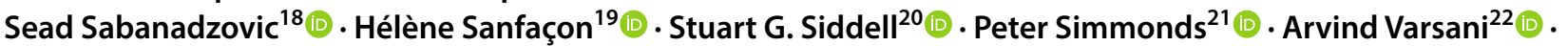 \\ Francisco Murilo Zerbini ${ }^{23}$ (D) . Andrew J. Davison ${ }^{24}$ (D)
}

Received: 11 April 2018 / Accepted: 14 April 2018 / Published online: 12 May 2018

(c) This is a U.S. government work and its text is not subject to copyright protection in the United States; however, its text may be subject to foreign copyright protection 2018

\begin{abstract}
This article lists the changes to virus taxonomy approved and ratified by the International Committee on Taxonomy of Viruses in February 2018. A total of 451 species, 69 genera, 11 subfamilies, 9 families and one new order were added to the taxonomy. The current totals at each taxonomic level now stand at 9 orders, 131 families, 46 subfamilies, 803 genera and 4853 species. A change was made to the International Code of Virus Classification and Nomenclature to allow the use of the names of people in taxon names under appropriate circumstances. An updated Master Species List incorporating the approved changes was released in March 2018 (https://talk.ictvonline.org/taxonomy/).
\end{abstract}

\section{Introduction}

Changes to virus taxonomy (the Universal Scheme of Virus Classification of the International Committee on Taxonomy of Viruses [ICTV]) take place annually and are the result of a multi-stage process. In accordance with ICTV Statutes (http://www.ictv.global/statutes.asp) and ICTV Executive Committee (EC) procedures, proposals undergo a review process involving input from the ICTV Study Groups (SGs) and Subcommittees (SCs), other interested virologists, and the EC. Proposals may thus be considered on more than one occasion before they are submitted for ratification by the ICTV membership. Proposals are presented for ratification by publication on the ICTV website (http://www.ictv.globa 1) followed by an electronic vote. The latest set of proposals approved by the EC was made available on the ICTV website in January 2017 (see http://ictv.global/proposals-2017 for all proposals combined into a single zip file, and use

Handling Editor: Tim Skern.

Arcady R. Mushegian

mushegian2@gmail.com

Extended author information available on the last page of the article the links provided in the References to access individual proposals). A list of proposals was then emailed on January 24, 2018 to the 160 members of ICTV, namely the EC Members, Life Members, ICTV Subcommittee Members (including the SG chairs) and ICTV National Representatives. Members were then requested to vote on whether to ratify the taxonomic proposals (voting closed on February $25,2018)$.

Since the processing of this year's proposals overlapped the end of the 2014-2017 EC tenure and the start of the 2017-2020 EC tenure, members of the both ECs constitute the authorship of this article.

\section{Changes to virus taxonomy: taxa and nomenclature}

Large number of changes were ratified by ICTV members (Table 1). Increase in the number of all taxa compared to the 2017 taxonomy was within the range of $9-11 \%$, except for the number of subfamilies, which grew by $31 \%$.

A summary of the individual proposals is provided in Table 2. Names of newly created taxa are in bold italic type. Names of existing taxa that have been moved or renamed are 
Table 1 Summary of taxonomic changes approved in February 2018

\begin{tabular}{llcccc}
\hline Change & Orders & Families & Subfamilies & Genera & Species \\
\hline New & 1 & 9 & 11 & 69 & 451 \\
Moved & 0 & 4 & 2 & 75 & 341 \\
Moved/renamed & 0 & 0 & 0 & 1 & 18 \\
Abolished & 0 & 0 & 0 & $1^{\mathrm{a}}$ & 2 \\
Totals $^{\mathrm{b}}$ & 9 & 131 & 46 & 803 & 4853 \\
\hline
\end{tabular}

${ }^{a}$ Existing genus (Tectivirus) split into two new genera, resulting in a net gain of one genus

${ }^{\mathrm{b}}$ Total numbers of taxa now recognized and shown in the ICTV Master Species List

shown in plain italic type. Each proposal is cited and listed in the References, to acknowledge the authors' efforts and to provide a link to each specific proposal on the ICTV website. These documents remain available for any who wish to see the full details of the proposals.

\section{Changes to the International Code of Virus Classification and Nomenclature}

ICTV has adopted one change to the Code, by ratifying Proposal 2017.003G.A.v2.ICVCN_Rule_3.11_Change. "Change ICVCN Rule 3.11 to permit, with limitations, the names of people to be recognised in names of taxa". As with many other proposals dealing with general approaches to virus taxonomy and nomenclature, this proposed change was a subject of a lively debate in the community, and was adopted by the overwhelming majority of the ICTV. This change also allowed the creation of two new taxa in the order Caudovirales, i.e., the family Ackermannviridae, to honor Hans-Wolfgang Ackermann (1936-2017), a former Life Member of the ICTV and Professor Emeritus at Université Laval, for his foundational contributions to the biology and taxonomy of prokaryotic viruses, and the subfamily Mccleskeyvirinae, to honor Charles Shelton McCleskey (1904-1984), a former Professor Emeritus at Louisiana State University, for his pioneering work on viruses of Leuconostoc bacteria.

\section{Conclusion}

All of the taxonomic proposals listed below were approved by the 87-95 affirming votes from the 96 members who voted (a return rate of about $60 \%$ ). The changes are now part of the official ICTV taxonomy. Several proposals that had been discussed at the 2017 EC meeting were not included in the ballot because they needed further development. Several other proposals were found, after completion of the ratification rate, to contain typographical errors in species' names. According to the current ICTV procedures, these errors will need to be corrected by additional proposals to be submitted to the 2018 EC meeting. An up-to-date list 


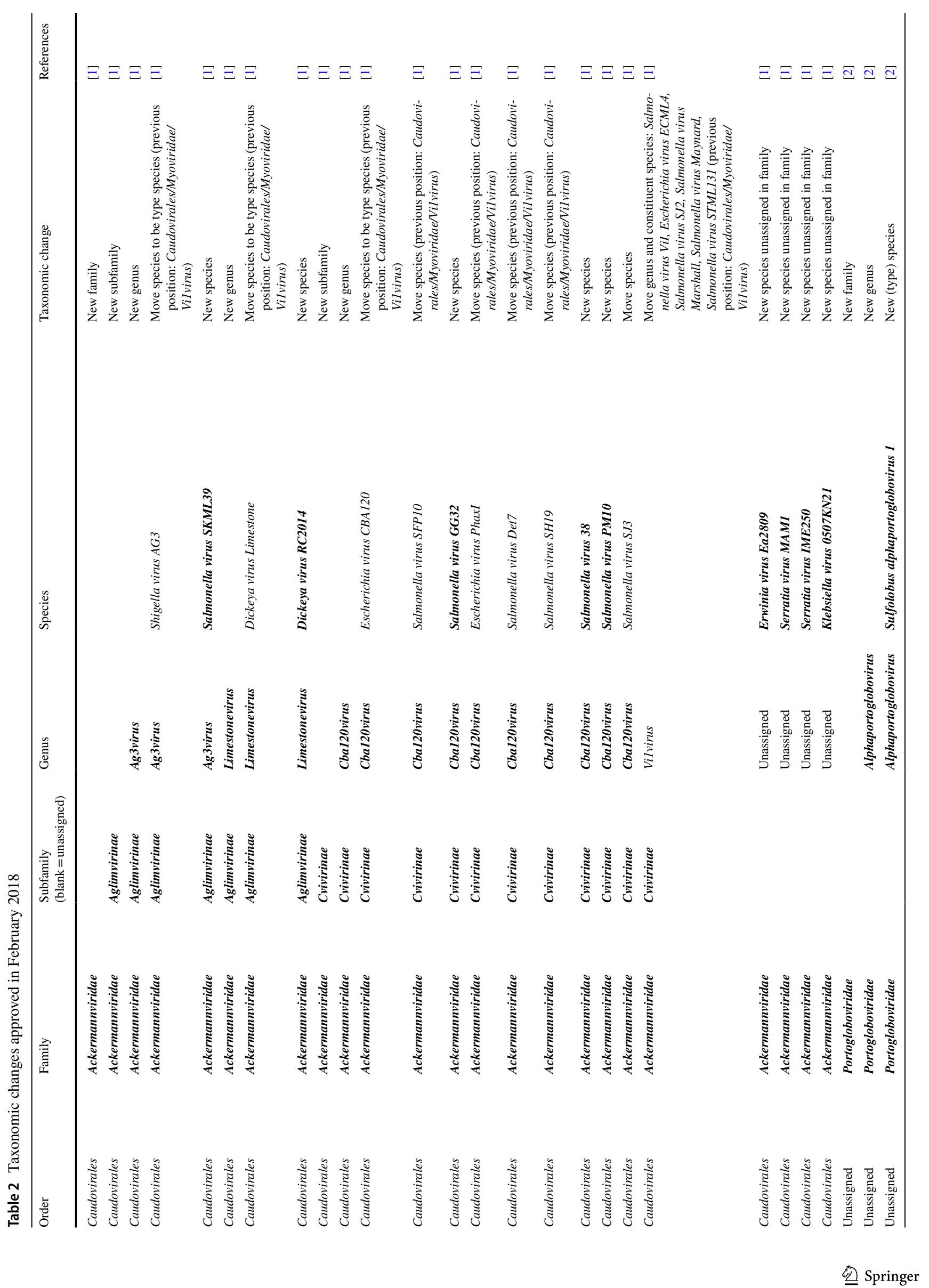




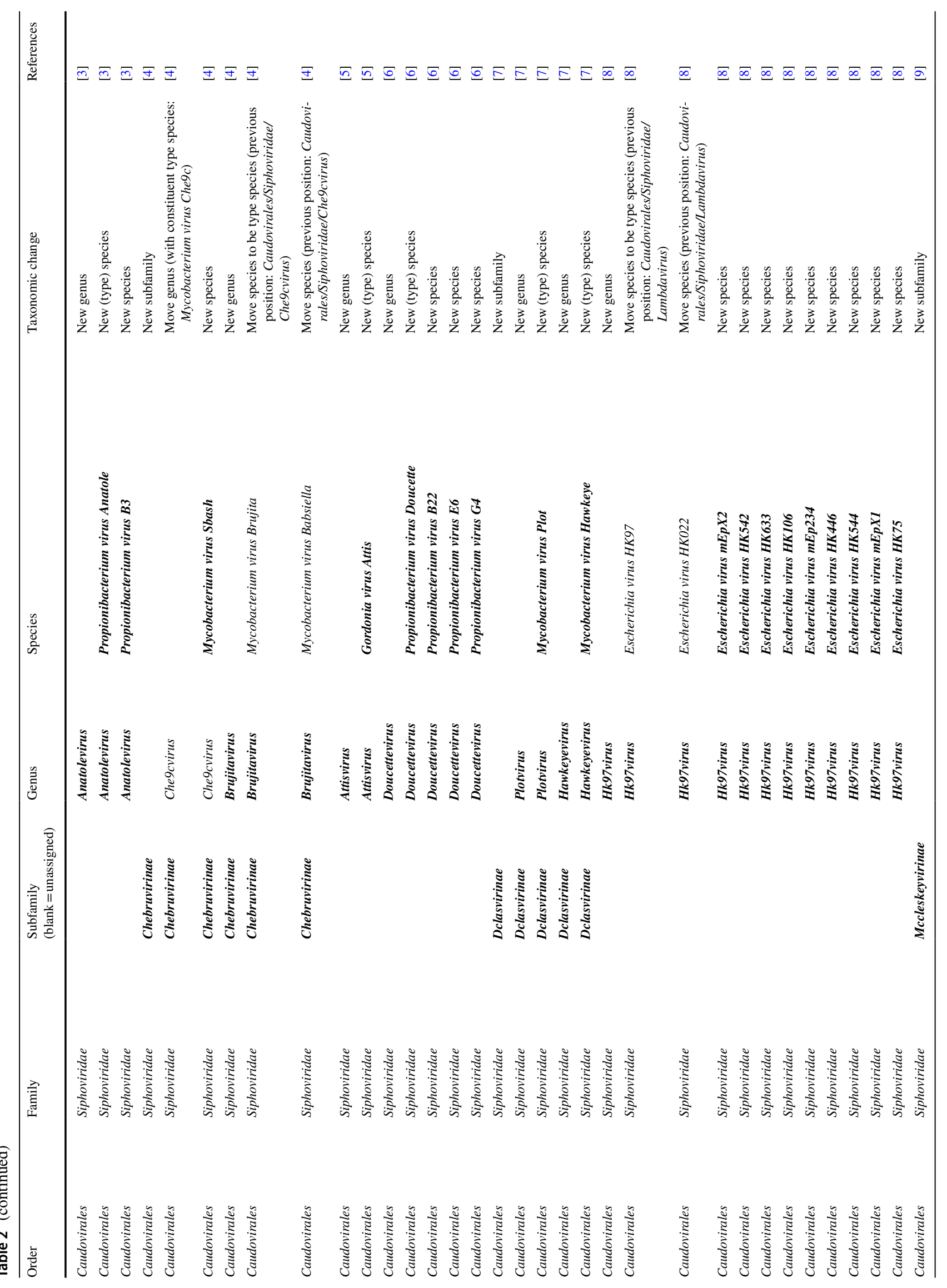




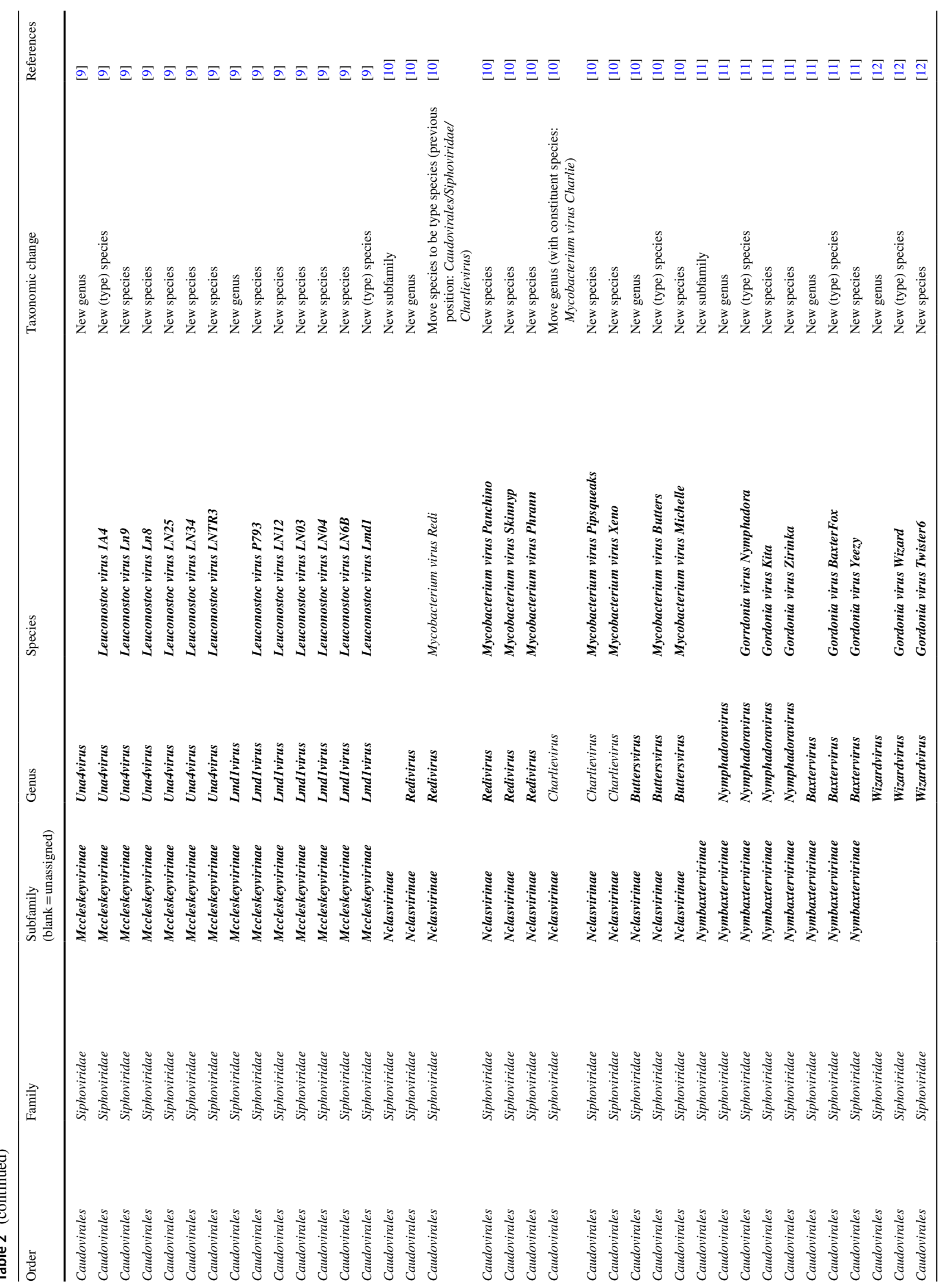




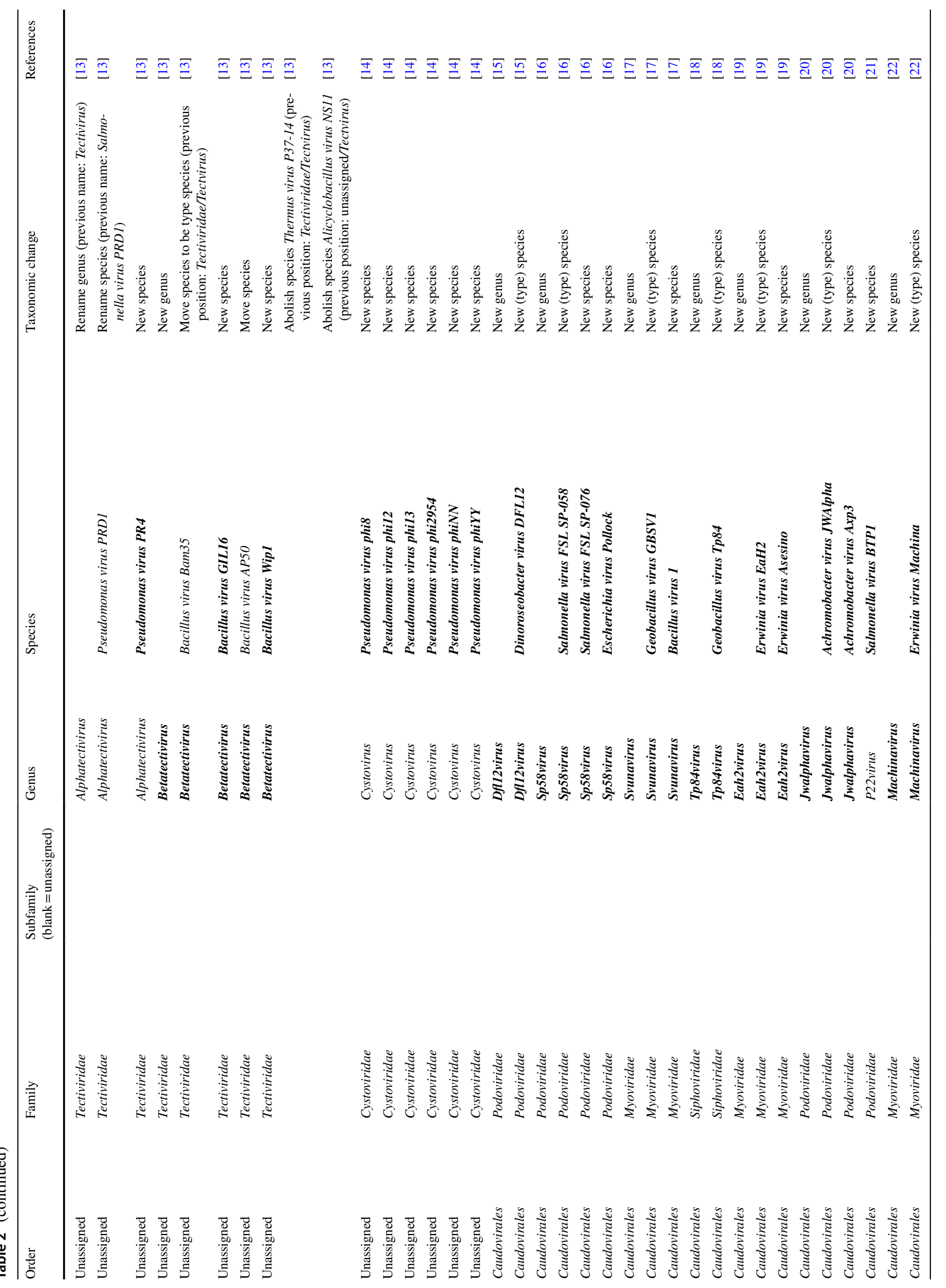




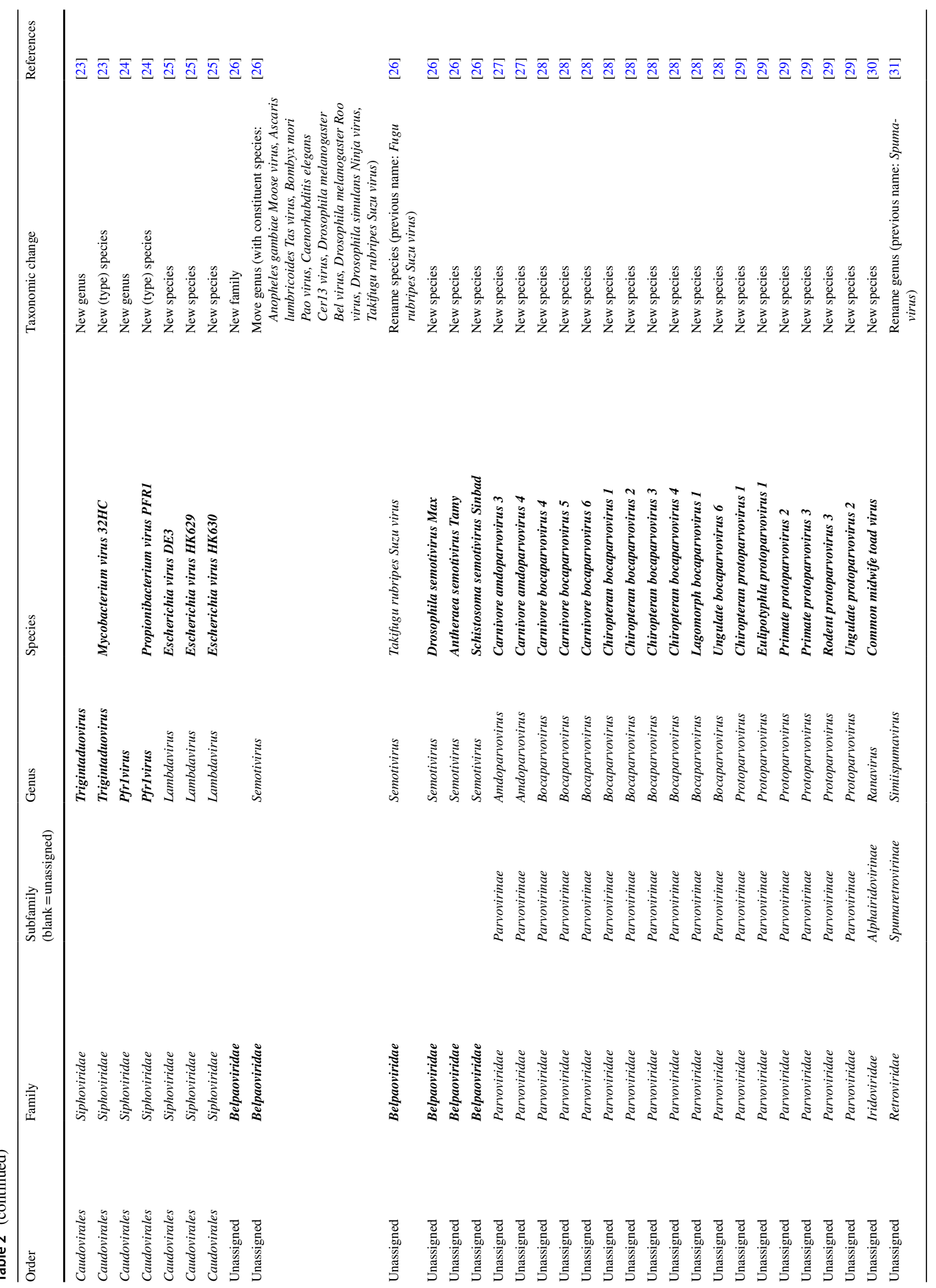




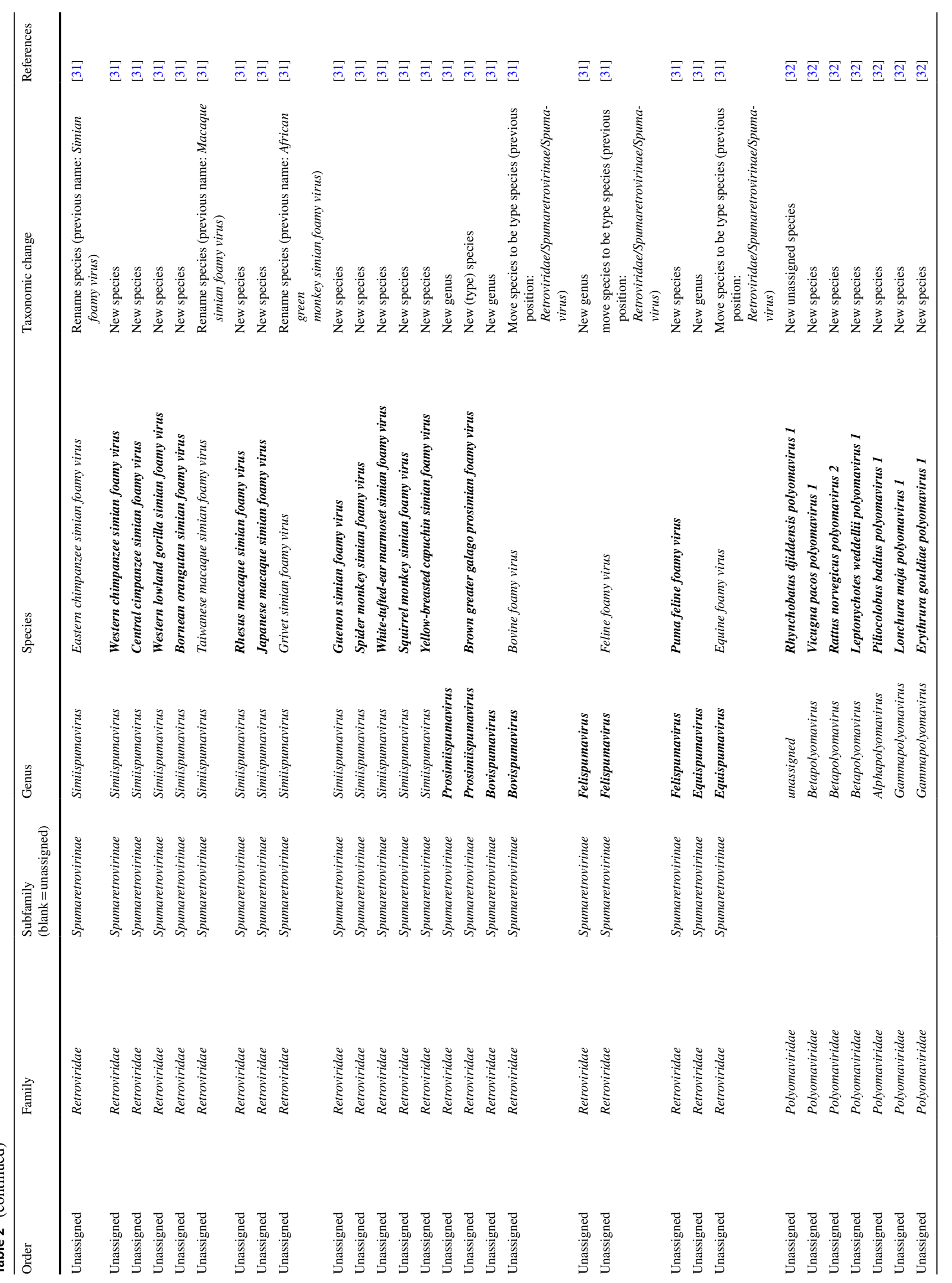




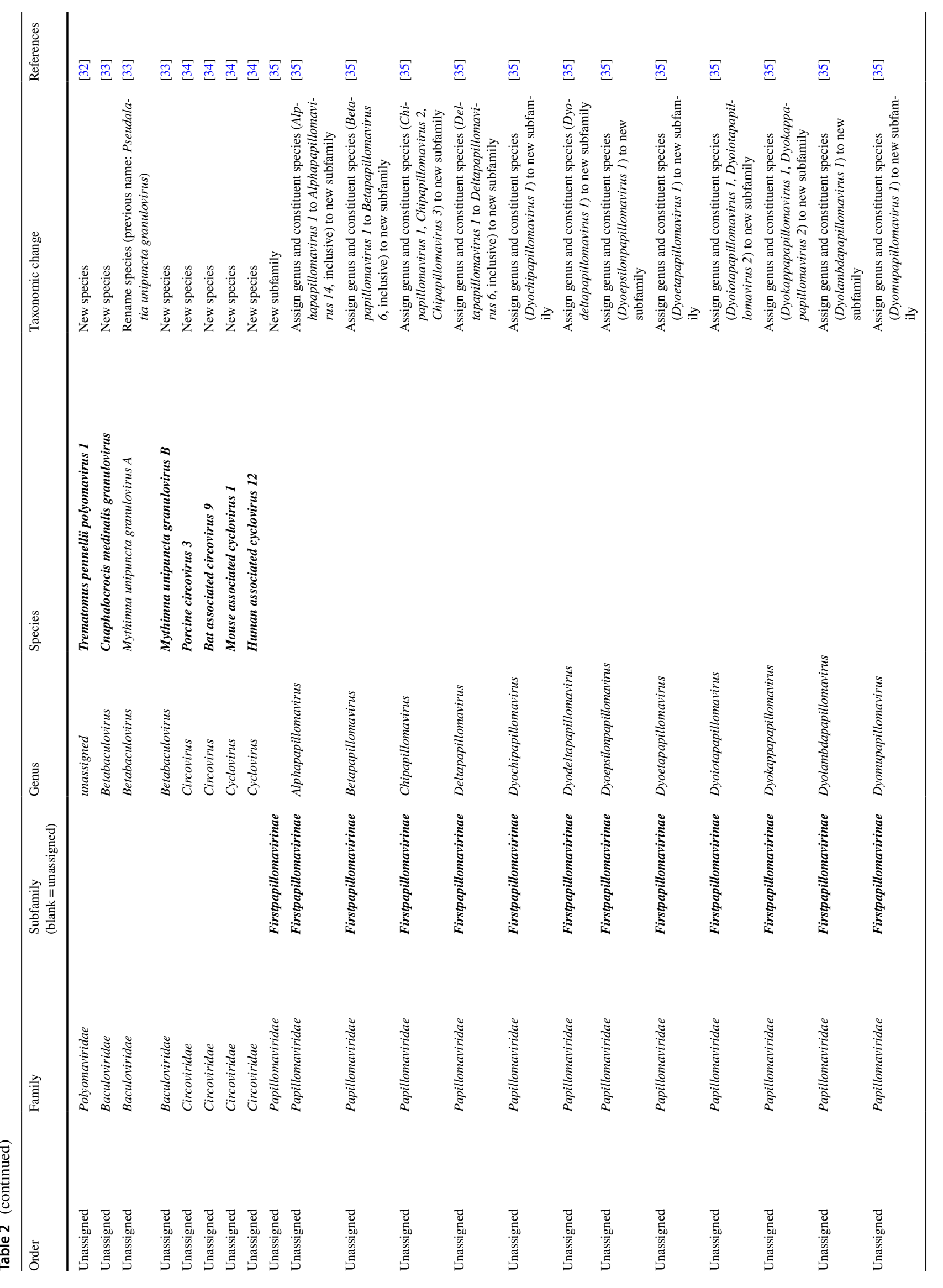




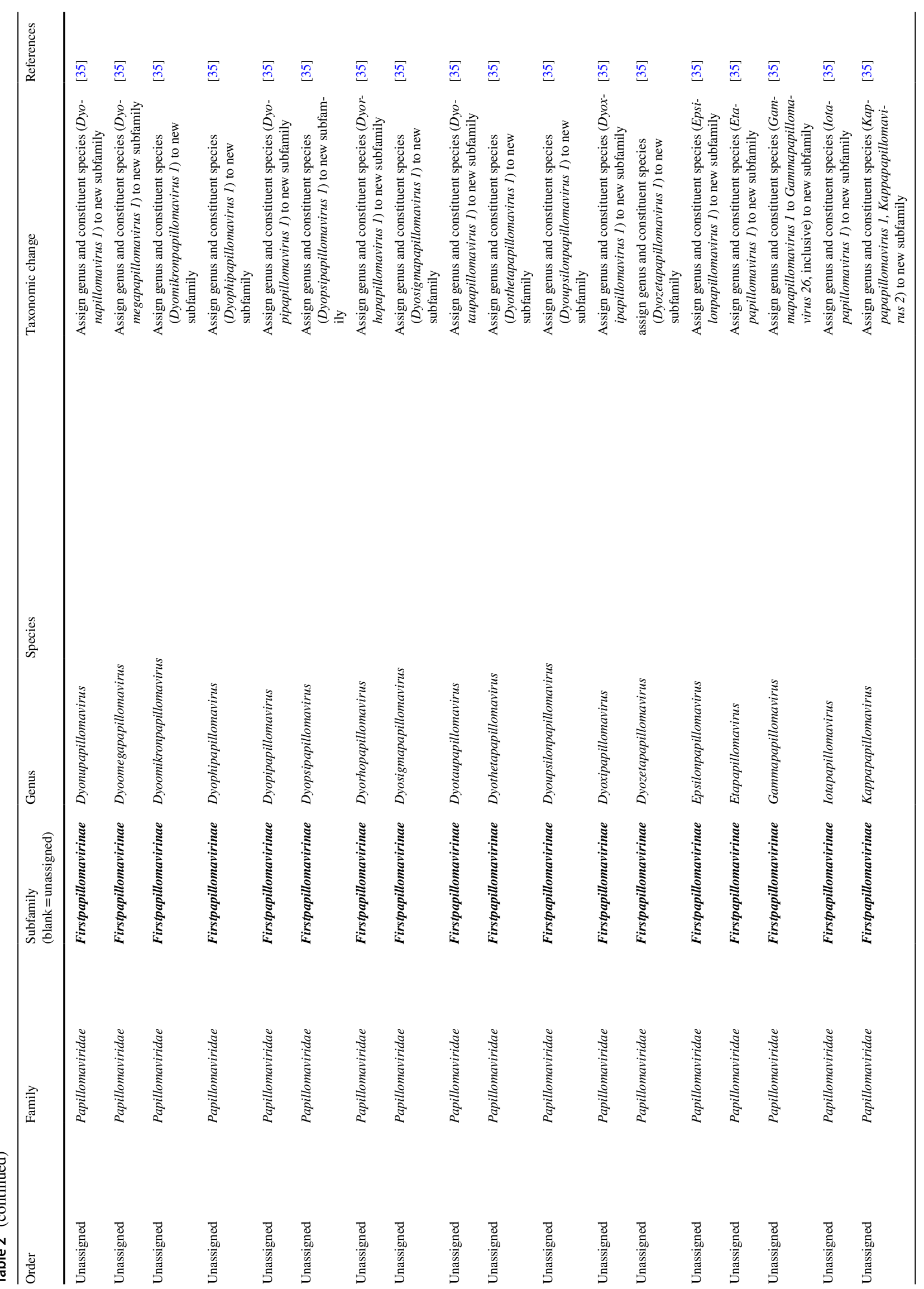




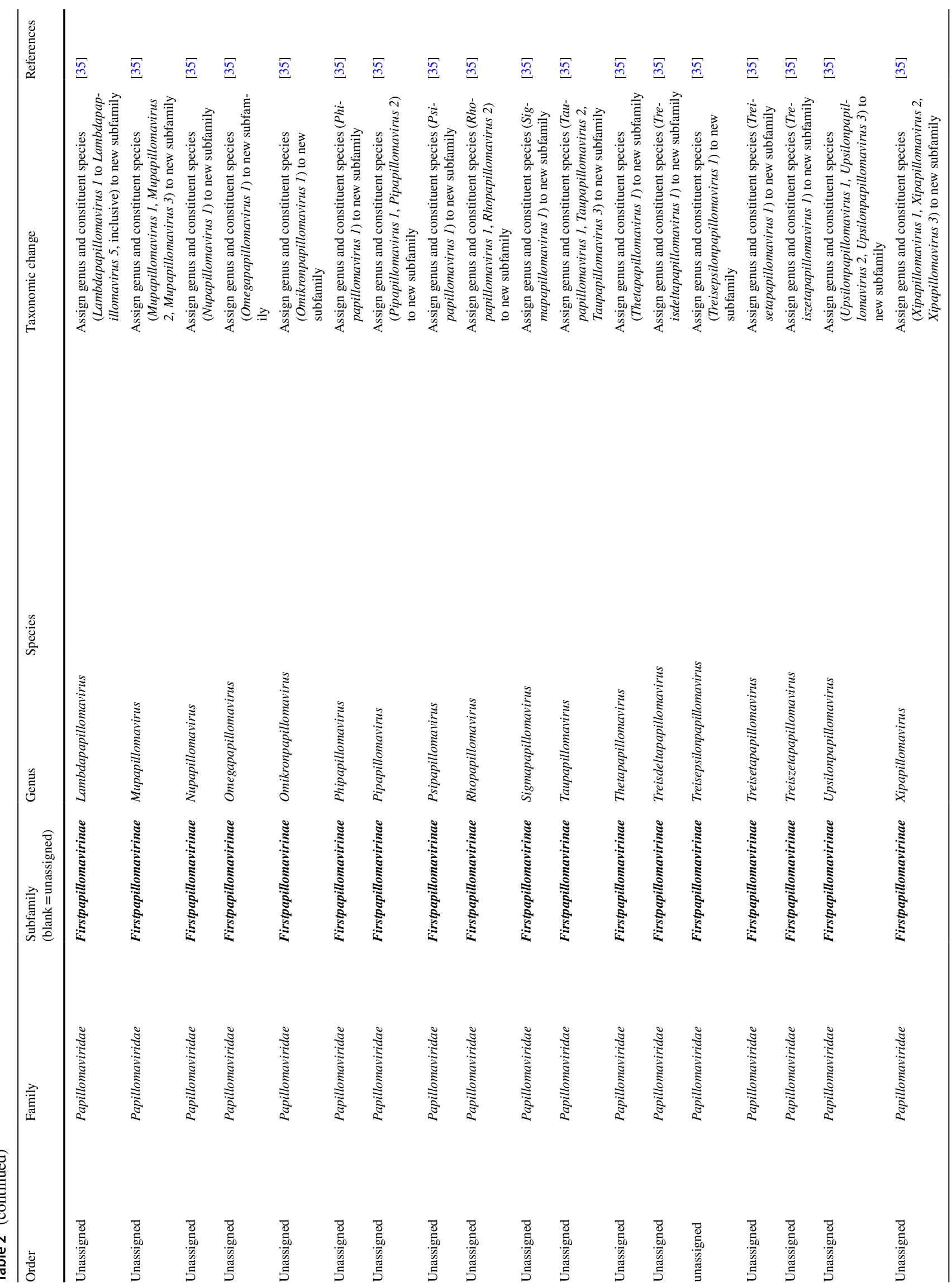




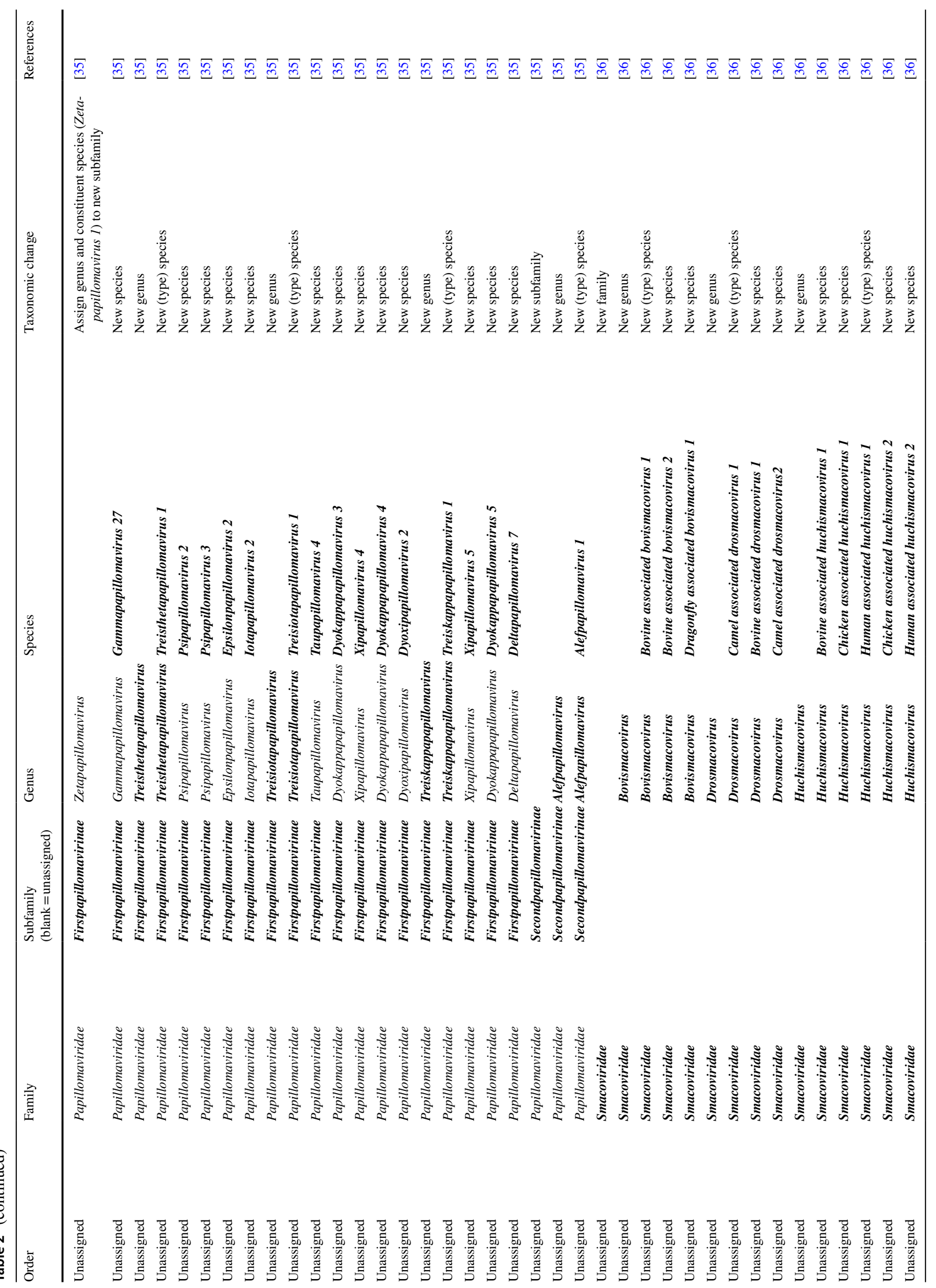




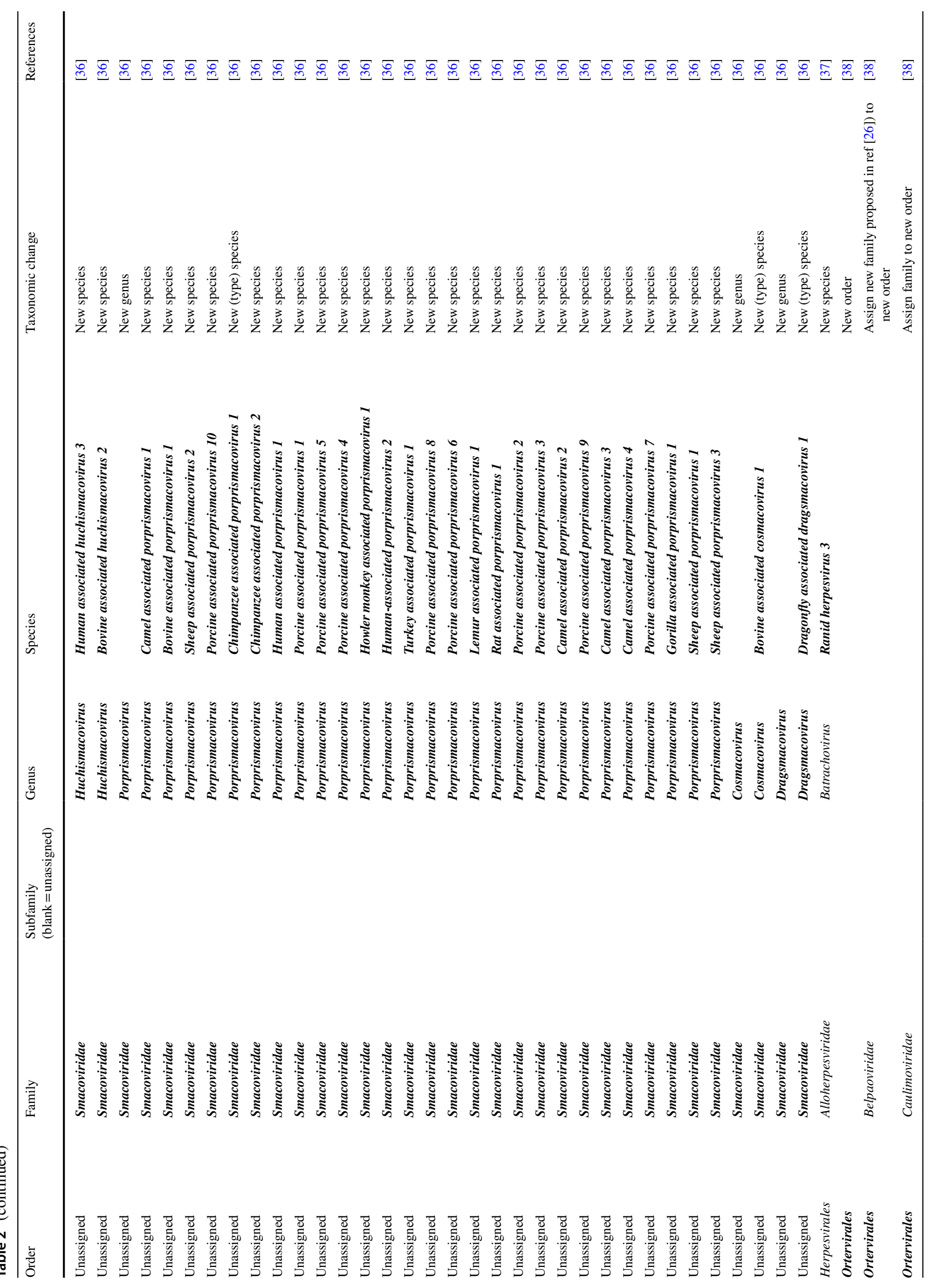




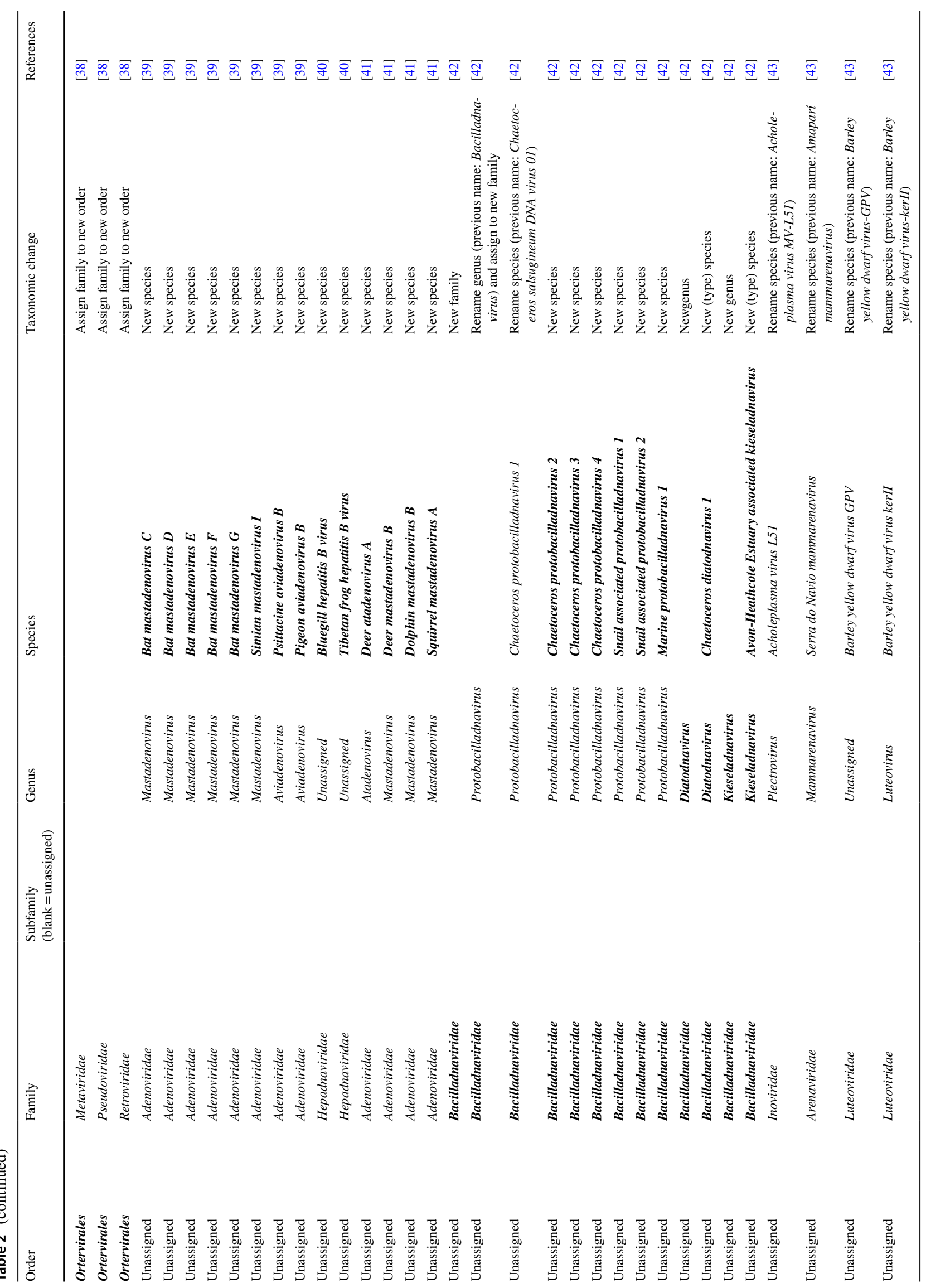




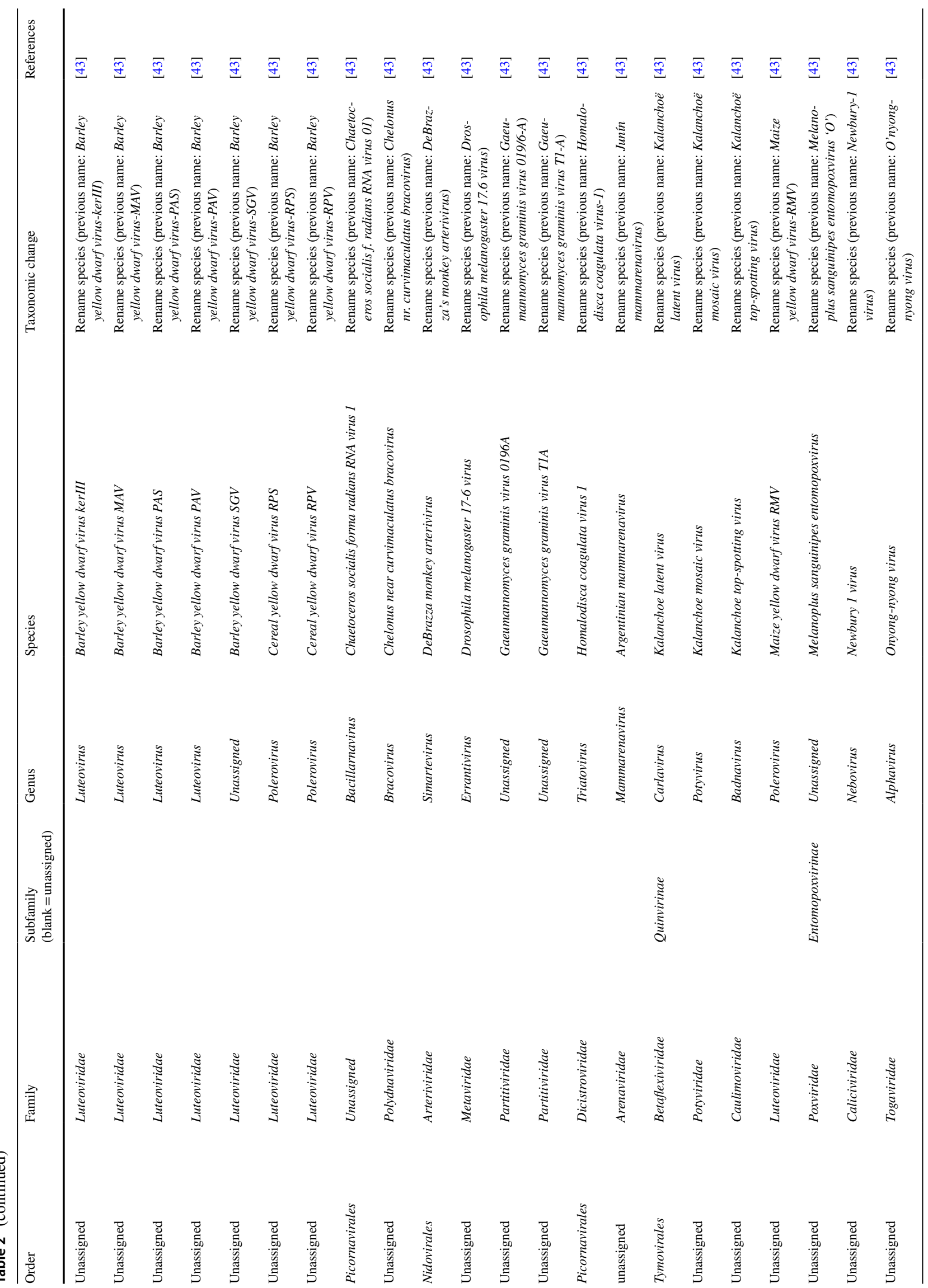




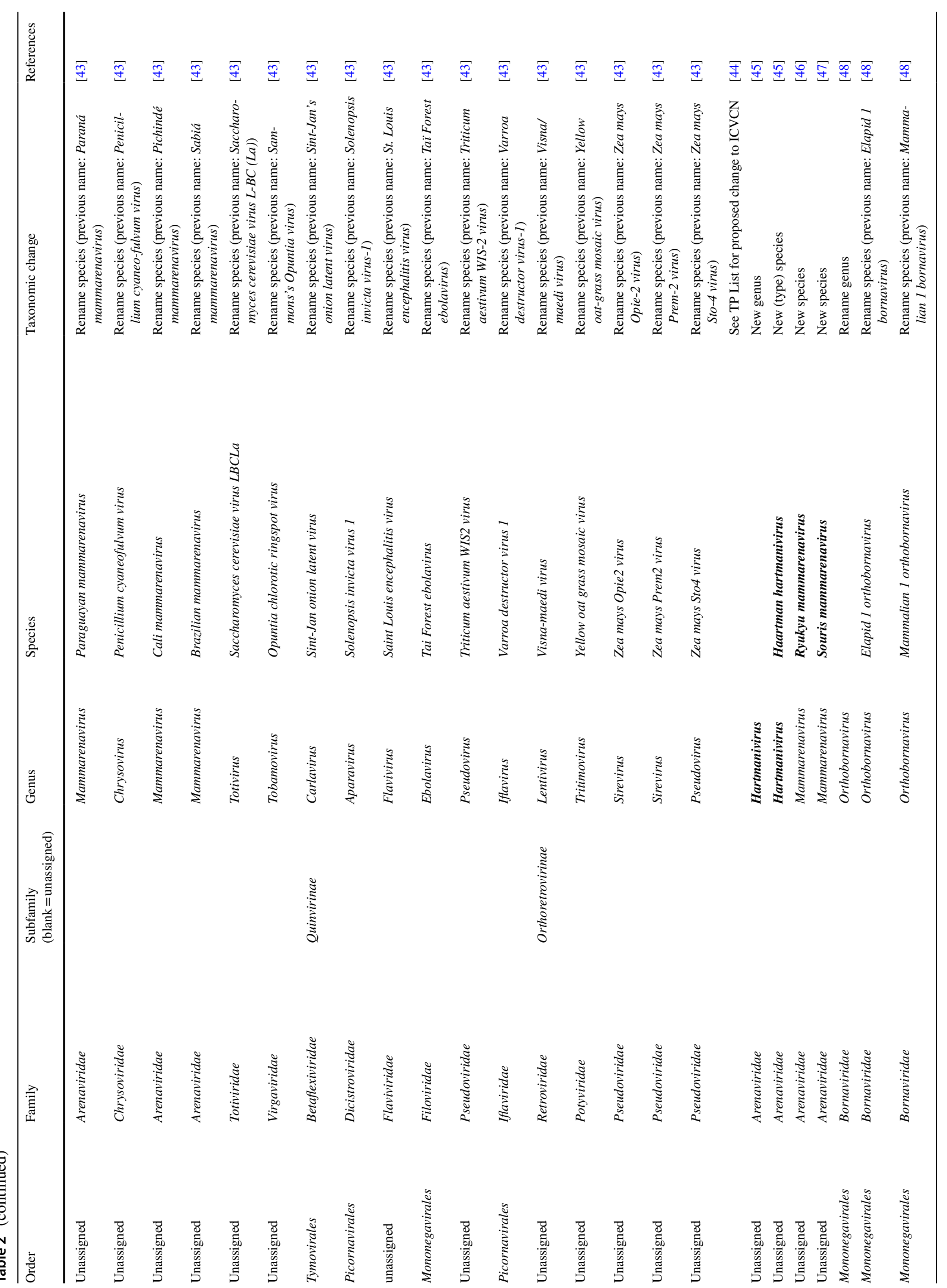




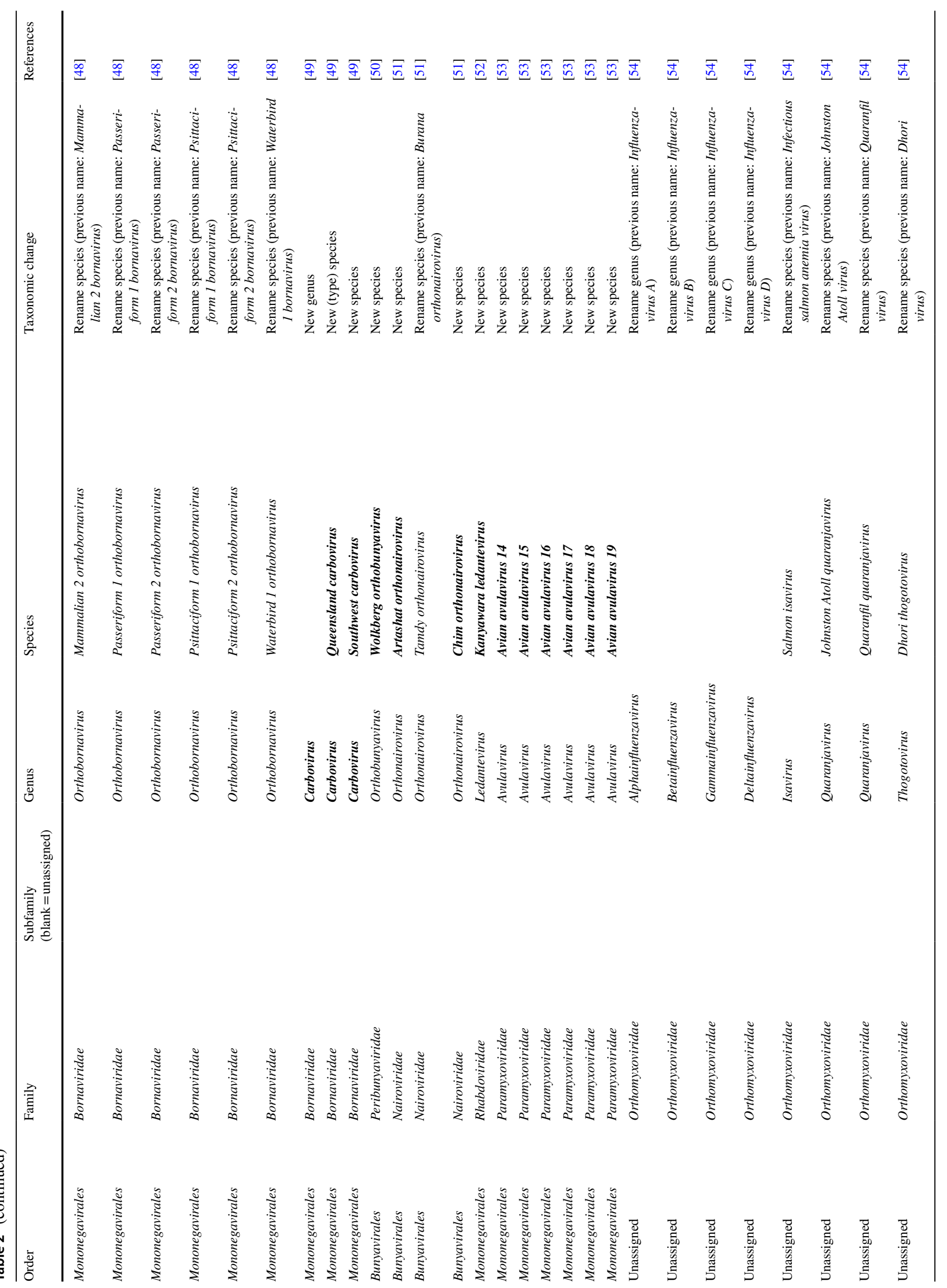




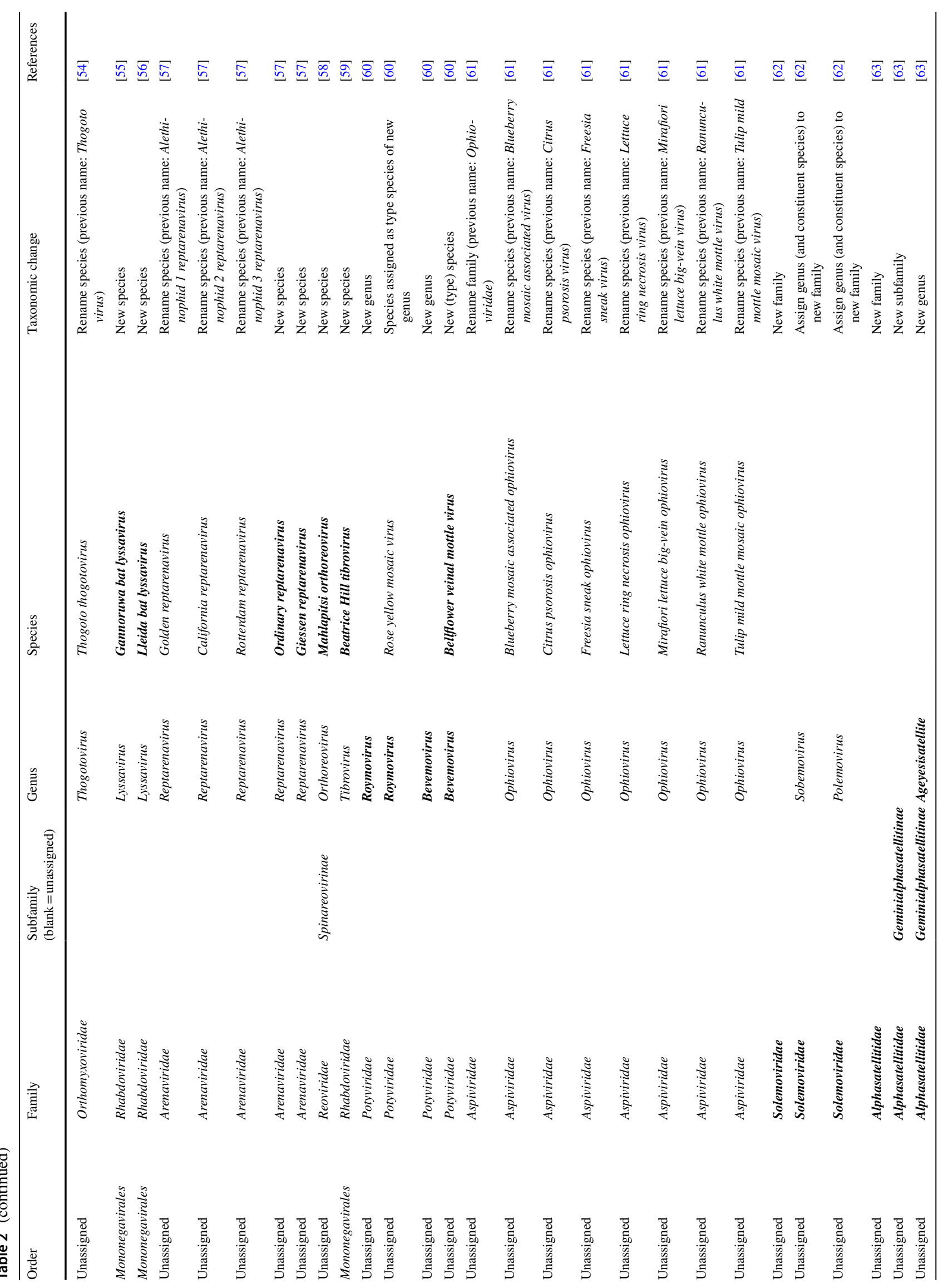




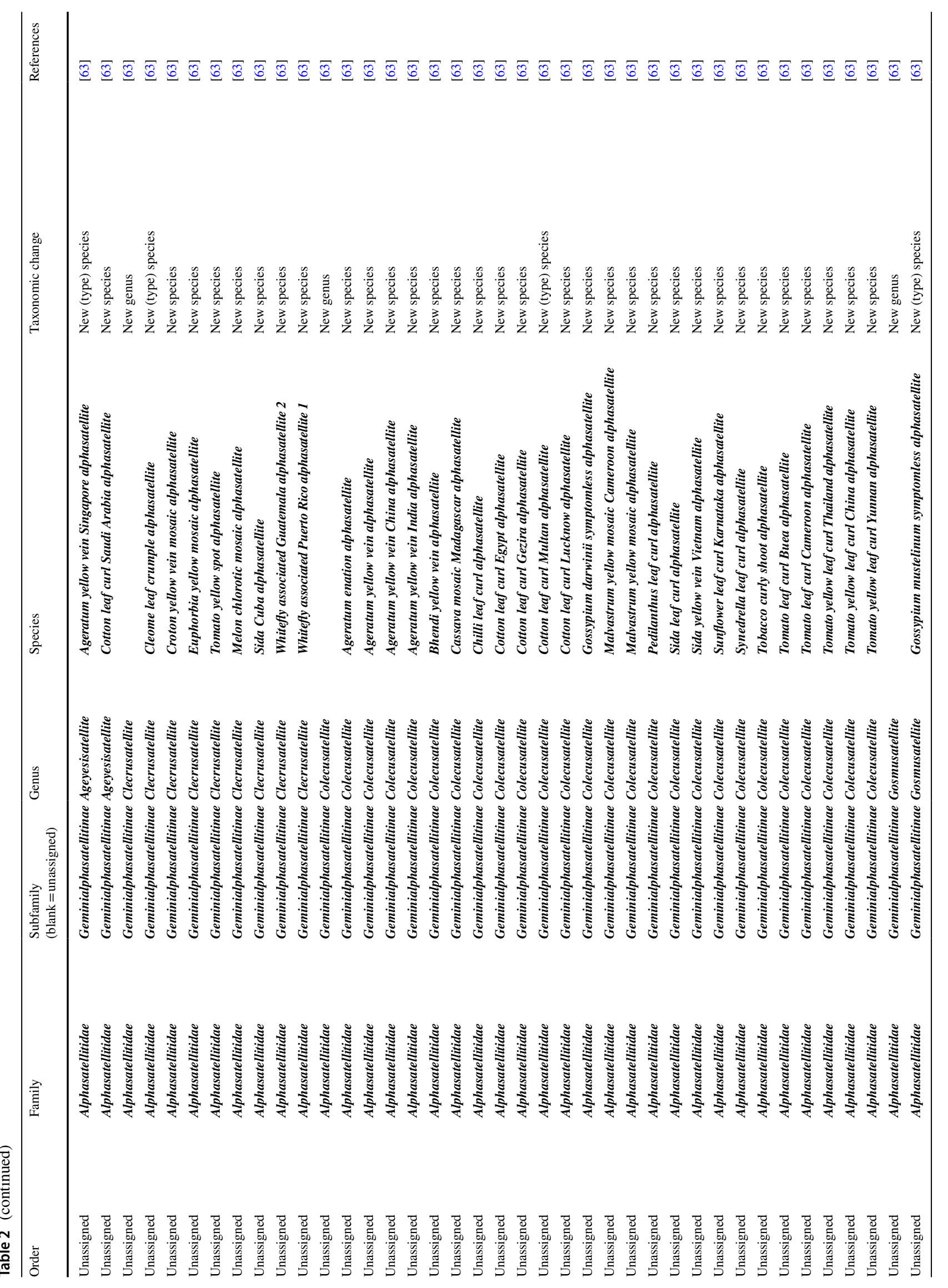




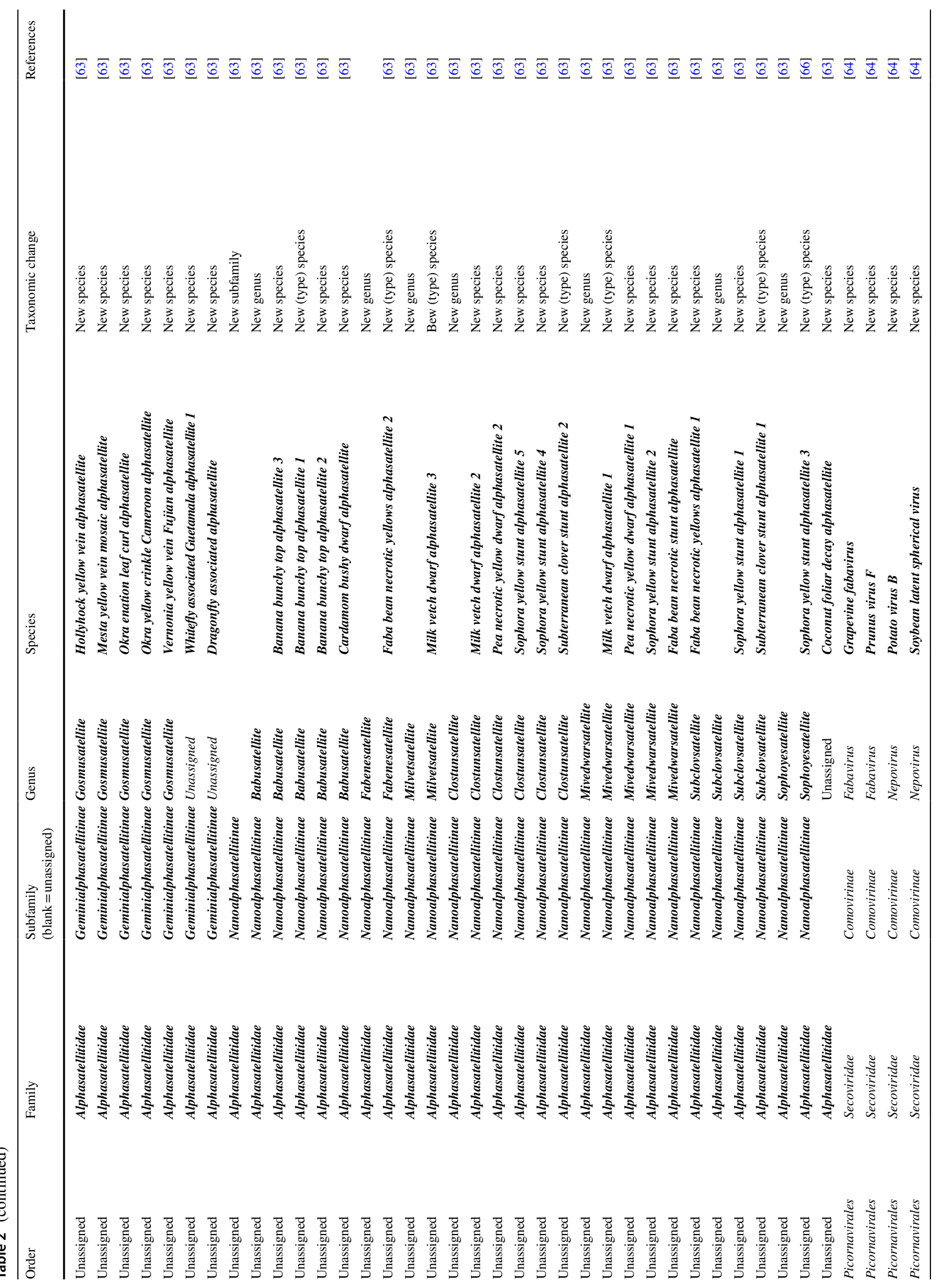




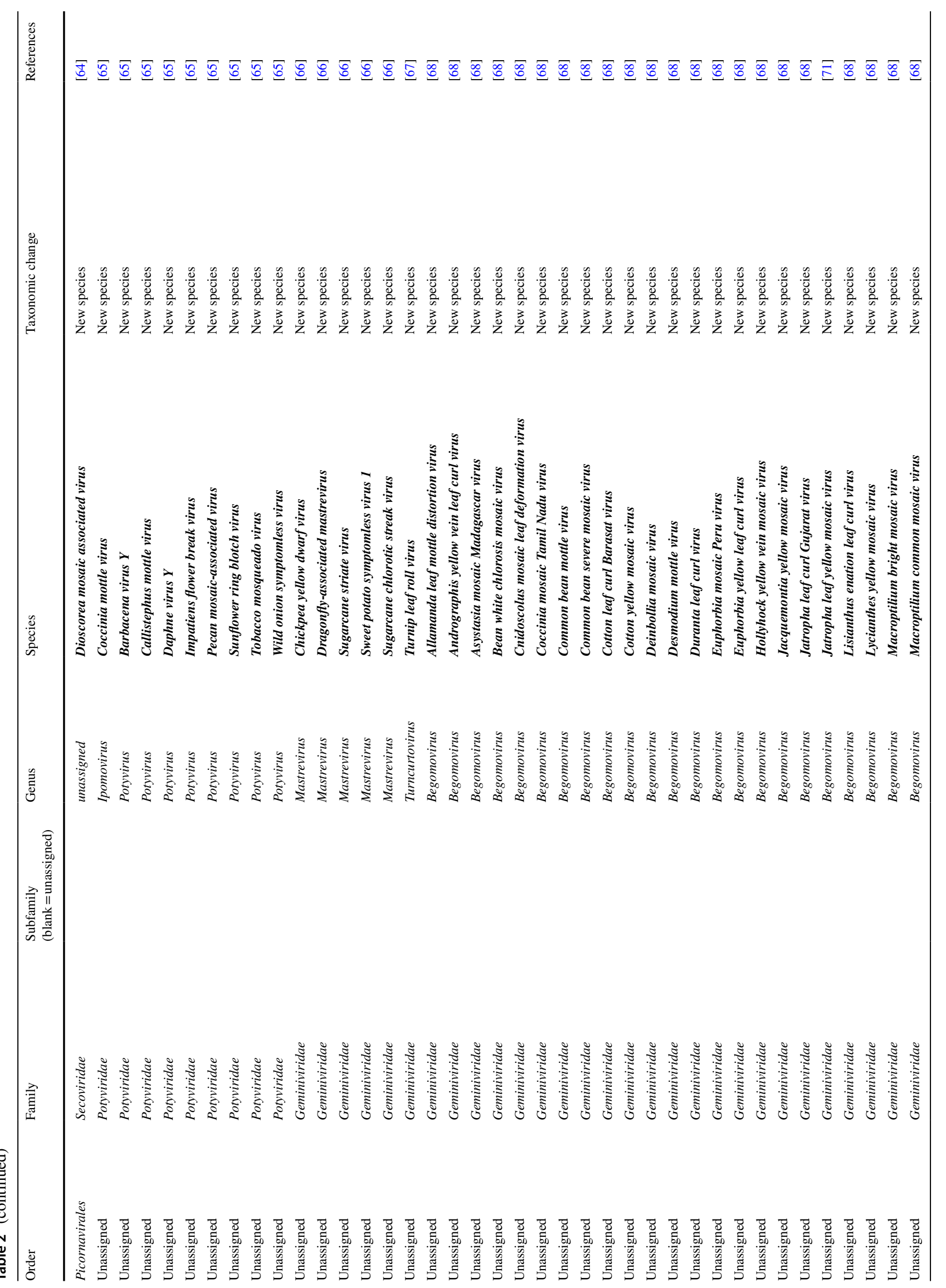




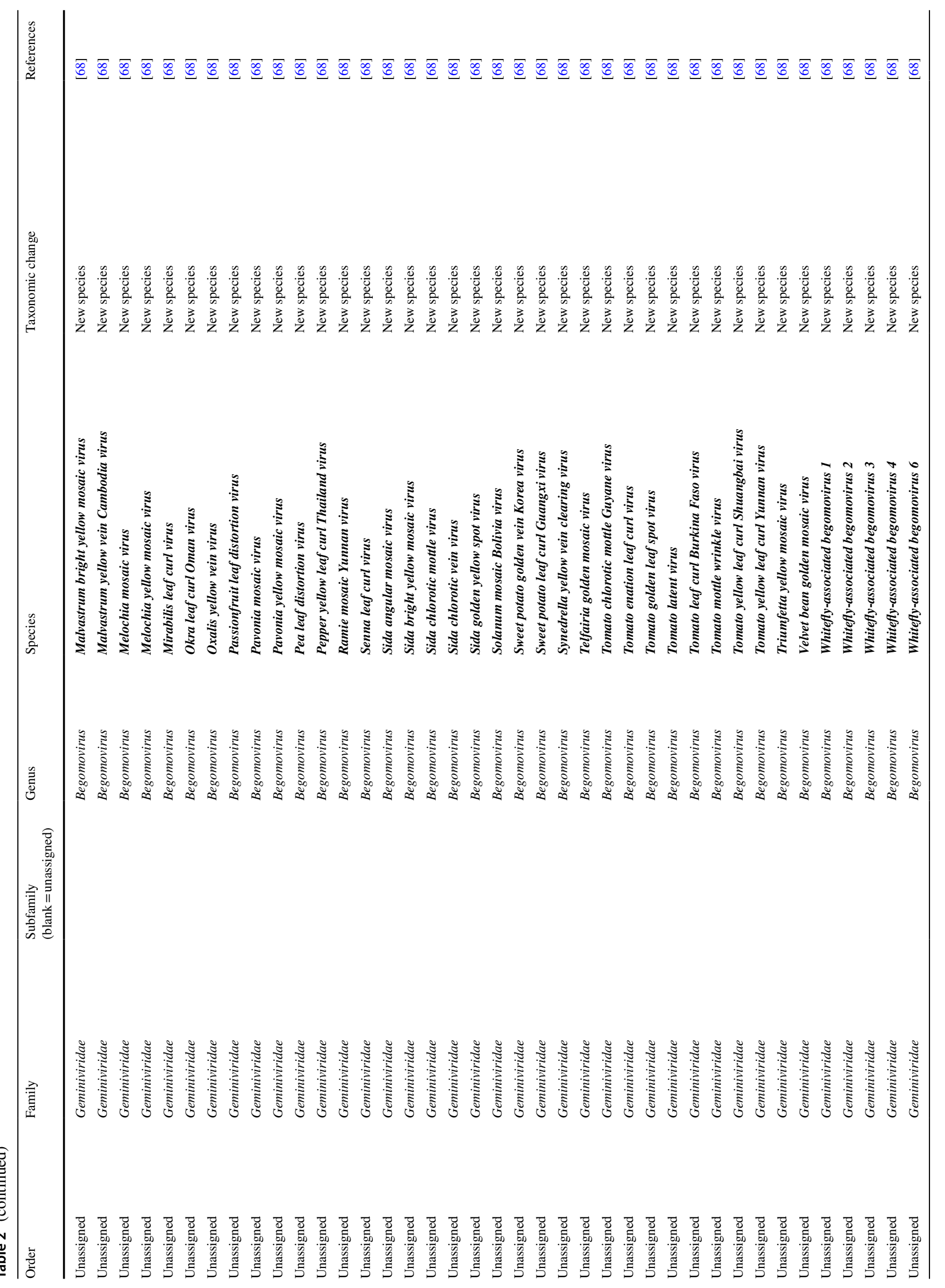




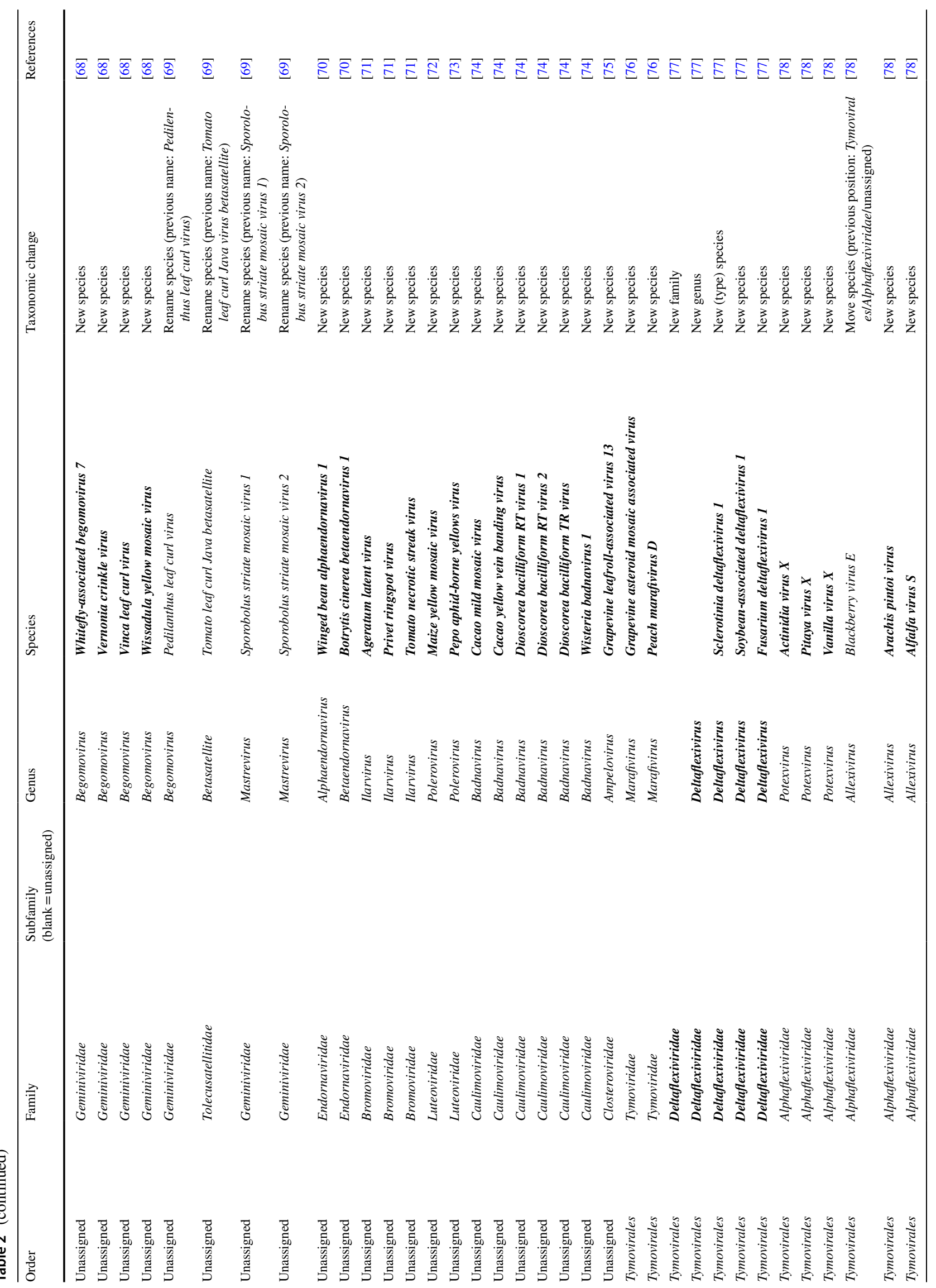




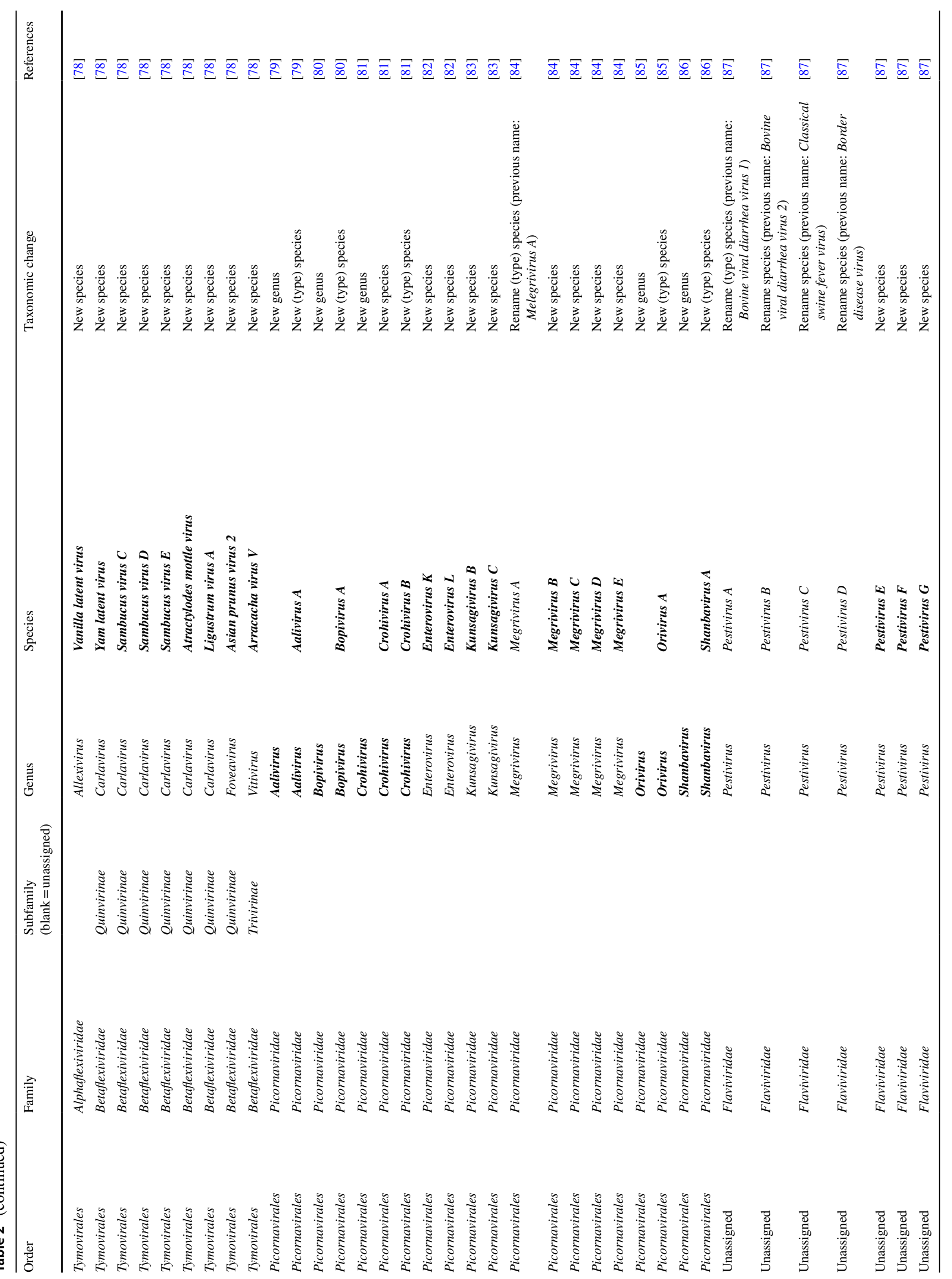




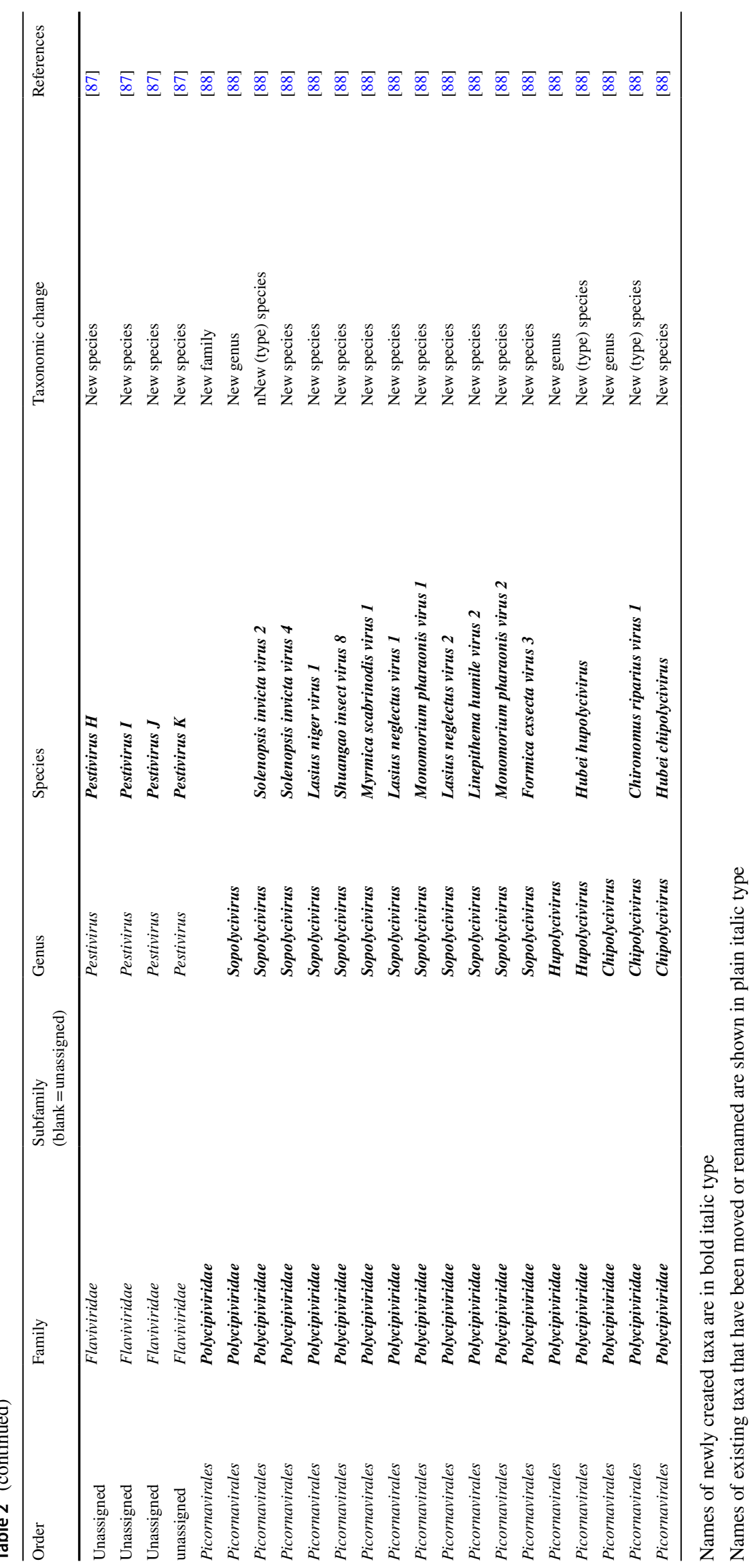


of all approved taxa can be found on the ICTV website at http://ictv.global/msl.

Acknowledgements A.J.D. is supported by the Medical Research Council (MC_UU_12014/3). B.E.D. is supported by Netherlands Organization for Scientific Research (NWO) Vidi Grant 864.14.004. A.E.G. is partially supported by EU project EVAg 653316 and the Leids Universiteits Fonds. N.J.K. is partially supported by core funding provided by the Biotechnology and Biological Sciences Research Council, UK. The content of this publication does not necessarily reflect the views or policies of the US Department of Health and Human Services or of the institutions and companies affiliated with the authors. This work was supported in part through Battelle Memorial Institute's prime contract with the US National Institute of Allergy and Infectious Diseases (NIAID) under Contract No. HHSN272200700016I (J.H.K). A.R.M. is a Program Director at the U.S. National Science Foundation (NSF); his work on this project was supported in part by the NSF Independent Research and Development program, but the statements and opinions expressed herein are made in a personal capacity and do not imply endorsement by NSF or the government of the United States. S.S. acknowledges financial support from Mississippi State University (MSU)—Mississippi Agricultural and Forestry Experiment Station (MAFES) Strategic Research Initiative grants.

\section{Compliance with ethical standards}

Conflict of interest The authors declare that they have no conflicts of interest.

Human rights This article does not contain any studies with human participants or animals performed by any of the authors. All authors have contributed to this work and agreed to its publication.

\section{References}

1. Kropinski AM, Anany H, Kuhn JH, Tolstoy I, Kutter E, Adriaenssens EM. Proposal 2017.001B.A.v3.Ackermannviridae. Create family Ackermannviridae in the order Caudovirales. http:// ictv.global/proposals-17/2017.001B.A.v3.Ackermannviridae.zip. Accessed 25 Apr 2018

2. Prangishvili D, Krupovic M. Proposal 2017.002B.A.v2.Portogloboviridae. Create a new genus, Alphaportoglobovirus, within a new family Portogloboviridae. http://ictv.global/proposals17/2017.002B.A.v2.Portogloboviridae.zip. Accessed 25 Apr 2018

3. Kropinski AM, Kuhn JH, Gillis A, Adriaenssens EM. Proposal 2017.003B.A.v1.Anetolevirus. Create genus Anetolevirus in family Siphoviridae, order Caudovirales. http://ictv.global/proposals17/2017.003B.A.v1.Anetolevirus.zip. Accessed 25 Apr 2018

4. Kropinski AM, Kuhn JH, Adriaenssens EM. Proposal 2017.004B.A.v1.Chebruvirinae. In family Siphoviridae create subfamily Chebruvirinae containing one existing and a new genus, Brujitavirus. http://ictv.global/proposals17/2017.004B.A.v1.Chebruvirinae.zip. Accessed 25 Apr 2018

5. Kropinski AM, Kuhn JH, Adriaenssens EM. Proposal 2017.005B.A.v1.Attisvirus. Create genus Attisvirus in family Siphoviridae, order Caudovirales. http://ictv.global/proposals17/2017.005B.A.v1.Attisvirus.zip. Accessed 25 Apr 2018

6. Kropinski AM, Kuhn JH, Adriaenssens EM. Proposal 2017.006B.A.v1.Doucettevirus. Create genus Doucettevirus in family Siphoviridae, order Caudovirales. http://ictv.global/propo sals-17/2017.006B.A.v1.Doucettevirus.zip. Accessed 25 Apr 2018
7. Morbidoni HR, Kropinski AM, Kuhn JH, Adriaenssens EM. Proposal 2017.007B.A.v1.Dclasvirinae. In family Siphoviridae create subfamily Dclasvirinae containing two new genera, Hawkeyevirus and Plotvirus. http://ictv.global/proposals17/2017.007B.A.v1.Dclasvirinae.zip. Accessed 25 Apr 2018

8. Kropinski AM, Kuhn JH, Adriaenssens EM, Refardt D. Proposal 2017.008B.A.v1.Hk97virus. Create genus Hk97virus in family Siphoviridae, order Caudovirales. http://ictv.global/proposals17/2017.008B.A.v1.Hk97virus.zip. Accessed 25 Apr 2018

9. Kropinski AM, Kuhn JH, Adriaenssens EM. Proposal 2017.009B.A.v1.Mccleskeyvirinae. In family Siphoviridae create subfamily Mccleskeyvirinae containing two new genera, Lmdlvirus and Una4virus. http://ictv.global/proposals17/2017.009B.A.v1.Mccleskeyvirinae.zip. Accessed 25 Apr 2018

10. Kropinski AM, Kuhn JH, Adriaenssens EM. Proposal 2017.010B.A.v2.Nclasvirinae. In family Siphoviridae create subfamily Nclasvirinae containing two new genera, Redivirus and Buttersvirus, and one existing genus. http://ictv.global/proposals17/2017.010B.A.v2.Nclasvirinae.zip. Accessed 25 Apr 2018

11. Kropinski AM, Kuhn JH, Adriaenssens EM. Proposal 2017.011B.A.v2.Nymbaxtervirinae. In family Siphoviridae create subfamily Nymbaxtervirinae containing two new genera, Nymphadoravirus and Baxtervirus. http://ictv.global/proposals17/2017.011B.A.v2.Nymbaxtervirinae.zip. Accessed 25 Apr 2018

12. Kropinski AM, Kuhn JH, Adriaenssens EM.2107.012B.A.v1.Wizardvirus. Create genus Wizardvirus in family Siphoviridae, order Caudovirales. http://ictv.global/proposals-17/2017.012B.A.v1. Wizardvirus.zip. Accessed 25 Apr 2018

13. Gillis A, Jang HB, Mahillon J, Kuhn JH, Kropinski AM, Adriaenssens EM, Lavigne R. Proposal 2017.013B.A.v2.Tectiviridae_rev. In the family Tectiviridae, change the name of genus Tectivirus to Alphatectivirus and create a new genus, Betatectivirus. http:// ictv.global/proposals-17/2017.013B.A.v2.Tectiviridae_rev.zip. Accessed 25 Apr 2018

14. Poranen MM, Mäntynen S, Kuhn JH, Adriaenssens EM, Kropinski AM. Proposal 2017.016B.A.v1.Cystovirus_6sp. Create six species in the genus Cystovirus, family Cystoviridae. http:// ictv.global/proposals-17/2017.016B.A.v1.Cystovirus_6sp.zip. Accessed 25 Apr 2018

15. Wittmann J, Kropinski AM, Kuhn JH, Adriaenssens EM. Proposal 2017.017B.A.v1.Dfl12virus. Create genus Dfl12virus in the family Podoviridae, order Caudovirales. http://ictv.global/propo sals-17/2017.017B.A.v1.Dfl12virus.zip. Accessed 25 Apr 2018

16. Wittmann J, Moreno Switt AI, Kropinski AM, Kuhn JH, Adriaenssens EM. Proposal 2017.018B.A.v1.Sp58virus. Create genus Sp58virus in the family Podoviridae, order Caudovirales. http://ictv.global/proposals-17/2017.018B.A.v1.Sp58virus.zip. Accessed 25 Apr 2018

17. Kropinski AM, Kuhn JH, Adriaenssens EM. Proposal 2017.019B.A.v1.Svunavirus. Create genus Svunavirus in the family Podoviridae, order Caudovirales. http://ictv.global/propo sals-17/2017.019B.A.v1.Svunavirus.zip. Accessed 25 Apr 2018

18. Skowron P, Kropinski AM, Kuhn JH, Adriaenssens EM, Los N. Proposal 2017.020B.A.v1.Tp84virus. Create genus Tp84virus within the family Siphoviridae, order Caudovirales. http://ictv. global/proposals-17/2017.020B.A.v1.Tp84virus.zip. Accessed 25 Apr 2018

19. Adriaenssens EM, Kuhn JH, Kropinski AM, Hope S, Grose JH. Proposal 2017.021B.A.v1.Eah2virus. Create genus Eah2virus within the family Myoviridae, order Caudovirales. http://ictv. global/proposals-17/2017.021B.A.v1.Eah2virus.zip. Accessed 25 Apr 2018

20. Wittmann J, Kropinski AM, Rohde M, Kuhn JH, Adriaenssens EM. Proposal 2017.022B.A.v2.Jwalphavirus. Create genus Jwalphavirus in the family Podoviridae, order Caudovirales. http:// 
ictv.global/proposals-17/2017.022B.A.v2.Jwalphavirus.zip. Accessed 25 Apr 2018

21. Owen V, Hinton JCD, Kropinski AM, Kuhn JH, Adriaenssens EM. Proposal 2017.023B.A.v1.P22virus_sp. Create a species in genus P22virus in the family Podoviridae, order Caudovirales. http://ictv.global/proposals-17/2017.023B.A.v1.P22virus_sp.zip. Accessed 25 Apr 2018

22. Adriaenssens EM, Kuhn JH, Kropinski AM, Hope S, Grose JH. Proposal 2017.024B.A.v1.Machinavirus. Create genus Machinavirus in family Myoviridae, order Caudovirales. http://ictv.globa 1/proposals-17/2017.024B.A.v1.Machinavirus.zip. Accessed 25 Apr 2018

23. Morbidoni HR, Kropinski AM, Kuhn JH, Adriaenssens EM. Proposal 2017.026B.A.v2.Trigintaduovirus. Create genus Trigintaduovirus in the family Siphoviridae, order Caudovirales. http:// ictv.global/proposals-17/2017.026B.A.v2.Trigintaduovirus.zip. Accessed 25 Apr 2018

24. Petrovski S, Kropinski AM , Kuhn JH, Adriaenssens EM. Proposal 2017.027B.A.v1.Pfr1virus. Create genus Pfrlvirus in the family Siphoviridae, order Caudovirales. http://ictv.global/propo sals-17/2017.027B.A.v1.Pfr1virus.zip. Accessed 25 Apr 2018

25. Kropinski AM, Adriaenssens EM. Proposal 2017.028B.A.v1. Lambdavirus_3sp. Create three species in the genus Lambdavirus, family Siphoviridae, order Caudovirales. http://ictv.global/propo sals-17/2017.028B.A.v1.Lambdavirus_3sp.zip. Accessed 25 Apr 2018

26. Krupovic M, Llorens C, Koonin EV, Kuhn JH. Proposal 2017.001D.A.v1.Belpaoviridae. Create 3 species within genus Semotivirus and move genus from family Metaviridae to a new family Belpaoviridae. http://ictv.global/proposals17/2017.001D.A.v1.Belpaoviridae.zip. Accessed 25 Apr 2018

27. Canuti M, McDonald E, Lang AS. Proposal 2017.002D.A.v1. Amdoparvovirus_2sp. Create 2 species in the genus Amdoparvovirus, subfamily Parvovirinae, family Parvoviridae. http://ictv. global/proposals-17/2017.002D.A.v1.Amdoparvovirus_2sp.zip. Accessed 25 Apr 2018

28. Cotmore SF, Agbandje-McKenna M, Canuti M, Chiorini JA, Eis-Hubinger A-M, Hughes J, Modha S, Ogliastro M, Pintel DJ, Qiu J, Soderlund-Venermo M, Tattersall P, Tijssen P. Proposal 2017.003D.A.v2.Bocaparvovirus_9sp. Create 9 species in the genus Bocaparvovirus, subfamily Parvovirinae, family Parvoviridae. http://ictv.global/proposals-17/2017.003D.A.v2.Bocap arvovirus_9sp.zip. Accessed 25 Apr 2018

29. Cotmore SF, Agbandje-McKenna M, Canuti M, Chiorini JA, Eis-Hubinger A-M, Hughes J, Modha S, Ogliastro M, Pintel DJ, Qiu J, Soderlund-Venermo M, Tattersall P, Tijssen P. Proposal 2017.004D.A.v2.Protoparvovirus_6sp. Create 6 species in the genus Protoparvovirus, subfamily Parvovirinae, family Parvoviridae. http://ictv.global/proposals-17/2017.004D.A.v2.Proto parvovirus_6sp.zip. Accessed 25 Apr 2018

30. Saucedo Garnica B, Rijks JM. Proposal 2017.005D.A.v1.Ranavirus_sp. Create a species in family Iridoviridae, genus Ranavirus, subfamily Alphairidovirinae. http://ictv.global/proposals17/2017.005D.A.v1.Ranavirus_sp.zip. Accessed 25 Apr 2018

31. Khan AS, Bodem J, Buseyne F, Gessain A, Johnson W, Kuhn JH, Kuzmak J, Lindemann D, Linial ML, Löchelt M, Materniak M, Soares MA, Switzer WM. Proposal 2017.006D.A.v2. Spumaretrovirinae_4gen. In the subfamily Spumaretrovirinae, create four genera (Bovispumavirus, Equispumavirus, Felispumavirus, Prosimiispumavirus) and change the names of genus Spumavirus to Semiispumavirus and its constituent species likewise. http://ictv.global/proposals-17/2017.006D.A.v2.Spumaretro virinae_4gen.zip. Accessed 25 Apr 2018

32. Calvignac-Spencer S, Daugherty MD, Feltkamp MCW, Lauber C, Moens U, Ramqvist T, Verschoor EJ, Ehlers B. Proposal 2017.007D.A.v2.Polyomaviridae_sp. Create 8 species within the family Polyomaviridae. http://ictv.global/proposals17/2017.007D.A.v2.Polyomaviridae_sp.zip. Accessed 25 Apr 2018

33. Harrison RL, Herniou EA, Krell PJ, Theilmann DA, Becnel JJ, Jehle JA, Burand JP. Proposal 2017.008D.A.v1. Betabaculovirus_2sp1ren. In genus Betabaculovirus (family Baculoviridae), create 2 species and rename one species. http:// ictv.global/proposals-17/2017.008D.A.v1.Betabaculovirus_2sp1r en.zip. Accessed 25 Apr 2018

34. Varsani A, Rosario K, Segalés J, Delwart E, Breitbart M. Proposal 2017.009D.A.v1.Circoviridae_4sp. Create two species in each of the genera Circovirus and Cyclovirus, family Circoviridae. http:// ictv.global/proposals-17/2017.009D.A.v1.Circoviridae_4sp.zip. Accessed 25 Apr 2018

35. Burk R, Chen Z. Proposal 2017.010D.A.v1. Papillomaviridae_2subf. Create 17 species, 4 genera and 2 subfamilies in the family Papillomaviridae. http://ictv.global/propo sals-17/2017.010D.A.v1.Papillomaviridae_2subf.zip. Accessed 25 Apr 2018

36. Varsani A, Krupovic M. Proposal 2017.011D.A.v3.Smacoviridae. Create the family Smacoviridae containing six genera and 43 species. http://ictv.global/proposals-17/2017.011D.A.v3.Smacovirid ae.zip. Accessed 25 Apr 2018

37. Origgi FC. Proposal 2017.012D.A.v1.Batrachovirus_sp. Create a species in the genus Batrachovirus, family Alloherpesviridae, order Herpesvirales. http://ictv.global/proposals17/2017.012D.A.v1.Batrachovirus_sp.zip. Accessed 25 Apr 2018

38. Krupovic M, Harrach B, Sabanadzovic S, Sanfaçon H, Koonin EV, Kuhn JH, Johnson W, Blomberg J, Coffin J, Fan H, Gifford R, Lindemann D, Mayer J, Stoye J, Tristem M, Teycheney P-Y, Dasgupta I, Geering A, Hull R, Kreuze JF, Lockhart B, Muller E, Olszewski N, Pappu H, Pooggin M, Richert-Pöggeler K, Schoelz JE, Seal S, Stavolone L. Proposal 2017.013D.A.v1.Ortervirales. Create the order Ortervirales comprising 5 families of reverse-transcribing viruses. http://ictv.global/proposals-17/2017.013D.A.v1.Orter virales.zip. Accessed 25 Apr 2018

39. Harrach B, Böszörményi KP, Benkő M. Proposal 2017.014D.A.v1. Adenoviridae_8sp. Create 8 species in the family Adenoviridae. http://ictv.global/proposals-17/2017.014D.A.v1.Adenovirid ae_8sp.zip. Accessed 25 Apr 2018

40. Magnius L, Norder H. Proposal 2017.015D.A.v1. Hepdnaviridae_2sp. Create 2 species to be unassigned in the family Hepadnaviridae. http://ictv.global/proposals17/2017.015D.A.v1.Hepdnaviridae_2sp.zip. Accessed 25 Apr 2018

41. Harrach B, Benkő M. Proposal 2017.016D.A.v1. Adenoviridae_4sp. In the family Adenoviridae, create 1 species in genus Atadenovirus and 3 species in genus Mastadenovirus http:// ictv.global/proposals-17/2017.016D.A.v1.Adenoviridae_4sp.zip. Accessed 25 Apr 2018

42. Krupovic M, Kazlauskas D, Varsani A. Proposal 2017.001F.A.v1. Bacilladnaviridae. Create two new genera within a new family Bacilladnaviridae; also rename an existing genus and move from unassigned to the new family. http://ictv.global/proposals17/2017.001F.A.v1.Bacilladnaviridae.zip. Accessed 25 Apr 2018

43. Siddell S. Proposal 2017.001G.A.v2.43spren. Change the names of 43 virus species to accord with ICVCN Code, Section 3-II, Rule 3.13 regarding the use of diacritical and other proscribed characters in taxon names. http://ictv.global/propo sals-17/2017.001G.A.v2.43spren.zip. Accessed 25 Apr 2018

44. Kropinski AM, Kuhn JH and 133 others. Proposal 2017.003G.A.v2.ICVCN_Rule_3.11_Change. Change ICVCN Rule 3.11 to permit, with limitations, the names of people to be recognised in names of taxa http://ictv.global/proposals17/2017.003G.A.v2.ICVCN_Rule_3.11_Change.zip. Accessed 25 Apr 2018 
45. Hepojoki J, Salmenperä P, Sironen T, Hetzel U, Korzyukov Y, Kipar A, Vapalahti O, Maes P, Buchmeier MJ, Charrel R, Clegg CS, de la Torre C, DeRisi JL, Emonet S, Gonzalez JP, Kuhn JH, Lukashevich IS, Peters CJ, Radoshitzky SR, Romanowski V, Salvato MS, Stenglein MD. Proposal 2017.001 M.A.v1.Hartmanivirus. Create genus Hartmanivirus in the family Arenaviridae. http://ictv.global/proposals-17/2017.001M.A.v1.Hartmanivirus. zip. Accessed 25 Apr 2018

46. Buchmeier MJ, Charrel R, Clegg CS, de la Torre C, DeRisi JL, Emonet S, Gonzalez JP, Kuhn JH, Lukashevich IS, Peters CJ, Radoshitzky SR, Romanowski V, Salvato MS, Stenglein MD. Proposal 2017.002 M.A.v3.Mammarenavirus_sp. Create species Ryukyu mammarenavirus in genus Mammarenavirus, family Arenaviridae. http://ictv.global/proposals-17/2017.002M.A.v3. Mammarenavirus_sp.zip. Accessed 25 Apr 2018

47. Ng TFF, Schneider BS, Gillis A, LeBreton M, Kondov NO, Coffey L, Wolfe ND, Delwart E, Buchmeier MJ, Charrel R, Clegg CS, de la Torre C, DeRisi JL, Emonet S, Gonzalez JP, Kuhn JH, Lukashevich IS, Peters CJ, Radoshitzky SR, Romanowski V, Salvato MS, Stenglein MD. Proposal 2017.003 M.A.v1.Mammarenavirus_sp. Create species Souris mammarenavirus in genus Mammarenavirus, family Arenaviridae. http://ictv.global/propo sals-17/2017.003M.A.v1.Mammarenavirus_sp.zip. Accessed 25 Apr 2018

48. Rubbenstroth D, Briese T, Dürrwald R, Horie M, Kuhn JH, Nowotny N, Payne S, Schwemmle M, Tomonaga K. Proposal 2017.004 M.A.v1.Bornaviridae_ren. Change the name of genus Bornavirus (family Bornaviridae, order Mononegavirales) to Orthobornavirus and the names of all its constituent species likewise. http://ictv.global/proposals-17/2017.004M.A.v1.Bornavirid ae_ren.zip. Accessed 25 Apr 2018

49. Kuhn JH, Stenglein M, Wellehan J, Rubbenstroth D, Briese T, Dürrwald R, Horie M, Nowotny N, Payne S, Schwemmle M, Tomonaga K, Hyndman T. Proposal 2017.005 M.A.v1.Carbovirus. Create genus Carbovirus in family Bornaviridae. http://ictv. global/proposals-17/2017.005M.A.v1.Carbovirus.zip. Accessed 25 Apr 2018

50. Jansen van Vuren P, Wiley MR, Palacios G, Storm N, Kuhn JH, Markotter W, Birkhead M, Kemp A, Paweska JT. Proposal 2017.007 M.A.v1.Orthobunyavirus_sp. Create a species in genus Orthobunyavirus, family Peribunyaviridae, order Bunyavirales. http://ictv.global/proposals-17/2017.007M.A.v1.Orthobunyavirus _sp.zip. Accessed 25 Apr 2018

51. Palacios G, Alkhovsky SV, Kuhn JH. Proposal 2017.008 M.A.v1. Orthonairovirus_2sp1ren. Create 2 species in genus Orthonairovirus (family Nairoviridae, order Bunyavirales) and rename one species http://ictv.global/proposals-17/2017.008M.A.v1.Ortho nairovirus_2sp1ren.zip. Accessed 25 Apr 2018

52. Goldberg TL, Bennett AJ, Kityo R, Kuhn JH, Chapman CA. Proposal 2017.009 M.A.v1.Ledantevirus_sp. Create a species in genus Ledantevirus, family Rhabdoviridae, order Mononegavirales. http://ictv.global/proposals-17/2017.009M.A.v1.Ledantevir us_sp.zip. Accessed 25 Apr 2018

53. Kuhn JH, Verdugo C, Ng TFF, Mor SK, Choi K-S, Thomazelli LM, Ramírez VMN. Proposal 2017.010 M.A.v2.Avulavirus_6sp. Create 6 species in genus Avulavirus, family Paramyxoviridae, order Mononegavirales. http://ictv.global/proposals17/2017.010M.A.v2.Avulavirus_6sp.zip. Accessed 25 Apr 2018

54. Smith GJD, Bahl J, Donis R, Hongo S, Kochs G, Lamb R, McCauley J, Palese P, Perez D, Presti R, Rimstad E. Proposal 2017.011 M.A.v2.Orthomyxoviridae_ren. Change the names of 4 genera and 5 species in the family Orthomyxoviridae. http://ictv. global/proposals-17/2017.011M.A.v2.Orthomyxoviridae_ren.zip. Accessed 25 Apr 2018

55. Marston DA, Mueller T, Freuling C, Banyard AC, Fooks AR. Proposal 2017.013 M.A.v1.Lyssavirus_sp. Create a species in genus
Lyssavirus, family Rhabdoviridae, order Mononegavirales. http:// ictv.global/proposals-17/2017.013M.A.v1.Lyssavirus_sp.zip. Accessed 25 Apr 2018

56. Marston DA, Echevarria J, Aréchiga N, Vázquez S, Mueller T, Freuling C, Banyard AC, Fooks AR. Proposal 2017.014 M.A.v1. Lyssavirus_sp. Create a species in genus Lyssavirus, family Rhabdoviridae, order Mononegavirales. http://ictv.global/proposals17/2017.014M.A.v1.Lyssavirus_sp.zip. Accessed 25 Apr 2018

57. Hepojoki J, Sironen T, Bao Y, Maes P, Buchmeier MJ, Charrel R, Clegg CS, de la Torre C, DeRisi JL, Emonet S, Gonzalez JP, Kuhn JH, Lukashevich IS, Peters CJ, Radoshitzky SR, Romanowski V, Salvato MS, Stenglein MD. Proposal 2017.015 M.A.v1. Reptarenavirus_2sp3ren. In the genus Reptarenavirus (family Arenaviridae), create two species and rename three species. http:// ictv.global/proposals-17/2017.015M.A.v1.Reptarenavirus_2sp3r en.zip. Accessed 25 Apr 2018

58. Jansen van Vuren P, Wiley MR, Palacios G, Storm N, McCulloch S, Markotter W, Birkhead M, Kemp A, Paweska JT. Proposal 2017.018 M.A.v1.Orthoreovirus_sp. Create a species in the genus Orthoreovirus, subfamily Spinareovirinae, family Reoviridae. http://ictv.global/proposals-17/2017.018M.A.v1.Orthoreovi rus_sp.zip. Accessed 25 Apr 2018

59. Wiley MR, Prieto, K, Blasdell KR, Caì Y, Campos Lawson C, Walker PJ, Chiu CY, Palacios G, Kuhn JH. Proposal 2017.019 M.A.v1.Tibrovirus_sp. Create a species in the genus Tibrovirus, family Rhabdoviridae, order Mononegavirales. http:// ictv.global/proposals-17/2017.019M.A.v1.Tibrovirus_sp.zip. Accessed 25 Apr 2018

60. Wylie S, Adams MJ, Chalam C, Kreuze JF, Lopez-Moya JJ, Ohshima K, Praveen S, Rabenstein F, Wang A, Zerbini FM. Proposal 2017.001P.A.v2.Potyviridae_2gen. Create 2 genera, Roymovirus and Bevemovirus, in the family Potyviridae. http://ictv.globa 1/proposals-17/2017.001P.A.v2.Potyviridae_2gen.zip. Accessed 25 Apr 2018

61. García ML, Dal Bó E, da Graça JV, Gago-Zachert S, Hammond J, Moreno P, Natsuaki T, Pallás V, Navarro JA, Reyes CA, Luna GR, Sasaya T, Tzanetakis IE, Vaira AM, Verbeek M, Kuhn JH. Proposal 2017.002P.A.v2.Ophioviridae_ren. Change the name of the family Ophioviridae to Aspiviridae and adopt nonlatinised binomial names for its constituent species. http://ictv.global/propo sals-17/2017.002P.A.v2.Ophioviridae_ren.zip. Accessed 25 Apr 2018

62. Sõmera M, Truve E, Hebrard E. Proposal 2017.003P.A.v2. Solemoviridae. Create family Sobemoviridae, combining the Sobemovirus and Polemovirus genera. http://ictv.global/proposals17/2017.003P.A.v2.Solemoviridae.zip. Accessed 25 Apr 2018

63. Briddon R, Varsani A Proposal 2017.004P.A.v4.Alphasatellitidae. Create the Alphasatellitidae, a family of satellites comprising 2 new subfamilies and 11 new genera. http://ictv.global/proposals17/2017.004P.A.v4.Alphasatellitidae.zip. Accessed 25 Apr 2018

64. Thompson JR, Karasev AV, Sanfacon H, Dasgupta I, Tzanetakis I, Petrzik K, Fuchs M, Yoshikawa N, van der Vlugt R, Wetzel T, Iwanami T. Proposal 2017.005P.A.v2.Secoviridae_5sp. In the family Secoviridae create two species in genus Nepovirus, two species in genus Fabavirus and one unassigned species. http:// ictv.global/proposals-17/2017.005P.A.v2.Secoviridae_5sp.zip. Accessed 25 Apr 2018

65. Wylie S, Adams MJ, Chalam C, Kreuze JF, Lopez-Moya JJ, Ohshima K, Praveen S, Rabenstein F, Wang A, Zerbini FM. Proposal 2017.007P.A.v1.Potyviridae_9sp. Create 9 species in the family Potyviridae. http://ictv.global/proposals17/2017.007P.A.v1.Potyviridae_9sp.zip. Accessed 25 Apr 2018

66. Varsani A, Martin DP. Proposal 2017.008P.A.v2.Mastrevirus_5sp. Create five species in the genus Mastrevirus, family Geminiviridae. http://ictv.global/proposals-17/2017.008P.A.v2.Mastreviru s_5sp.zip. Accessed 25 Apr 2018 
67. Varsani A, Martin DP. Proposal 2017.009P.A.v1.Turncurtovirus_sp. Create a species in the genus Turncurtovirus, family Geminiviridae. http://ictv.global/proposals-17/2017.009P.A.v1. Turncurtovirus_sp.zip. Accessed 25 Apr 2018

68. Zerbini FM, Varsani A, Navas-Castillo J, Moriones E, Martin D, Briddon R. Proposal 2017.010P.A.v1.Begomovirus_66sp. Create 66 species in the genus Begomovirus, family Geminiviridae. http://ictv.global/proposals-17/2017.010P.A.v1.Begomoviru s_66sp.zip. Accessed 25 Apr 2018

69. Zerbini FM. Proposal 2017.011P.A.v1.Gemini_sp_ren. Correct the names of three species in the family Geminiviridae and one species in the Tolecusatellitidae. http://ictv.global/proposals17/2017.011P.A.v1.Gemini_sp_ren.zip. Accessed 25 Apr 2018

70. Sabanadzovic S, Valverde RA, Okada R. Proposal 2017.012P.A.v1.Endornaviridae_2sp. Create two species in the family Endornaviridae. http://ictv.global/proposals17/2017.012P.A.v1.Endornaviridae_2sp.zip. Accessed 25 Apr 2018

71. Bujarski J, Gallitelli D, Reddy MK, Garcia-Arenal F, Wang A, Scott SW. Proposal 2017.013P.A.v2.Ilarvirus_3sp. Create 3 species in the genus Ilarvirus, family Bromoviridae. http://ictv.global/ proposals-17/2017.013P.A.v2.Ilarvirus_3sp.zip. Accessed 25 Apr 2018

72. Matthiesen R, Miller WA. Proposal 2017.014P.A.v1.Polerovirus_sp. Create species, Maize yellow mosaic virus, in the genus Polerovirus, family Luteoviridae. http://ictv.global/proposals17/2017.014P.A.v1.Polerovirus_sp.zip. Accessed 25 Apr 2018

73. Ibaba JD, Gubba A, Laing MD. Proposal 2017.015P.A.v1.Polerovirus_sp. Create species, Pepo aphid-borne yellows virus, in the genus Polerovirus, family Luteoviridae. http://ictv.global/propo sals-17/2017.015P.A.v1.Polerovirus_sp.zip. Accessed 25 Apr 2018

74. Geering A, Bömer M, Turaki AA, Seal SE, Teycheney P-Y. Proposal 2017.017P.A.v2.Badnavirus_6sp. Create 6 species in the genus Badnavirus, family Caulimoviridae. http://ictv.global/propo sals-17/2017.017P.A.v2.Badnavirus_6sp.zip. Accessed 25 Apr 2018

75. Fuchs M, Agranovsky AA, Bar-Joseph M, Candresse T, Dolja V, Livieratos I, Martelli GP, Maree H, Melzer MJ, Menzel W, Minafra A, Sabanadzovic S. Proposal 2017.018P.A.v1.Ampelovirus_sp. Create a species in the genus Ampelovirus, family Closteroviridae. http://ictv.global/proposals-17/2017.018P.A.v1.Ampel ovirus_sp.zip. Accessed 25 Apr 2018

76. Sabanadzovic S, Edwards M, Hammond R, Haenni A-L, Martelli GP, Dreher T. Proposal 2017.019P.A.v1.Marafivirus_2sp. Create two species in the genus Marafivirus, family Tymoviridae. http:// ictv.global/proposals-17/2017.019P.A.v1.Marafivirus_2sp.zip. Accessed 25 Apr 2018

77. Kreuze J, Adams MJ, Candresse T, Hammond J, Menzel W, Pearson M, Saldarelli P, Vaira A, Yoshikawa N. Proposal 2017.020P.A.v1.Deltaflexiviridae. Create 3 species in a new genus, Deltaflexivirus, in a new family, Deltaflexiviridae, in the order Tymovirales. http://ictv.global/proposals17/2017.020P.A.v1.Deltaflexiviridae.zip. Accessed 25 Apr 2018

78. Kreuze J, Adams MJ, Candresse T, Hammond J, Menzel W, Pearson M, Saldarelli P, Vaira AM, Yoshikawa N. Proposal 2017.021P.A.v3.Alpha-Betaflexiviridae_14sp. Create 14 new species in the Alphaflexiviridae and Betaflexiviridae families and assign the unassigned species Blackberry virus $E$ to the genus Allexivirus. http://ictv.global/proposals-17/2017.021P.A.v3.Alpha -Betaflexiviridae_14sp.zip. Accessed 25 Apr 2018

79. Zell R, Delwart E, Gorbalenya AE, Hovi T, King AMQ, Knowles NJ, Lindberg AM, Pallansch MA, Palmenberg AC, Reuter G, Simmonds P, Skern T, Stanway G, Yamashita T. Proposal 2017.002S.A.v1.Aalivirus. Create 1 new species
(Aalivirus A) in new genus (Aalivirus). http://ictv.global/propo sals-17/2017.002S.A.v1.Aalivirus.zip. Accessed 25 Apr 2018

80. Zell R, Delwart E, Gorbalenya AE, Hovi T, King AMQ, Knowles NJ, Lindberg AM, Pallansch MA, Palmenberg AC, Reuter G, Simmonds P, Skern T, Stanway G, Yamashita T. Proposal 2017.003S.A.v1.Bopivirus. Create 1 new species (Bopivirus $A$ ) in a new genus (Bopivirus). http://ictv.global/proposals17/2017.003S.A.v1.Bopivirus.zip. Accessed 25 Apr 2018

81. Zell R, Delwart E, Gorbalenya AE, Hovi T, King AMQ, Knowles NJ, Lindberg AM, Pallansch MA, Palmenberg AC, Reuter G, Simmonds P, Skern T, Stanway G, Yamashita T. Proposal 2017.004S.A.v2.Crohivirus. Create 2 new species (Crohivirus A, Crohivirus $B$ ) in a new genus (Crohivirus). http://ictv.global/propo sals-17/2017.004S.A.v2.Crohivirus.zip. Accessed 25 Apr 2018

82. Zell R, Delwart E, Gorbalenya AE, Hovi T, King AMQ, Knowles NJ, Lindberg AM, Pallansch MA, Palmenberg AC, Reuter G, Simmonds P, Skern T, Stanway G, Yamashita T. Proposal 2017.005S.A.v1.Enterovirus_2sp. Create 2 new species, Enterovirus $K$, Enterovirus $L$ in the genus Enterovirus. http://ictv.globa 1/proposals-17/2017.005S.A.v1.Enterovirus_2sp.zip. Accessed 25 Apr 2018

83. Zell R, Delwart E, Gorbalenya AE, Hovi T, King AMQ, Knowles NJ, Lindberg AM, Pallansch MA, Palmenberg AC, Reuter G, Simmonds P, Skern T, Stanway G, Yamashita T. Proposal 2017.006S.A.v2.Kunsagivirus_2sp. Create 2 new species (Kunsagivirus $B$ \& Kunsagivirus $C$ ) in the genus Kunsagivirus . http:// ictv.global/proposals-17/2017.006S.A.v2.Kunsagivirus_2sp.zip. Accessed 25 Apr 2018

84. Zell R, Delwart E, Gorbalenya AE, Hovi T, King AMQ, Knowles NJ, Lindberg AM, Pallansch MA, Palmenberg AC, Reuter G, Simmonds P, Skern T, Stanway G, Yamashita T. Proposal 2017.007S.A.v2.Megrivirus_4sp. Change the name of species Melegrivirus A to MegrivirusA and create 4 new species (Megrivirus $B, C, D, E)$ in the genus Megrivirus. http://ictv.global/propo sals-17/2017.007S.A.v2.Megrivirus_4sp.zip. Accessed 25 Apr 2018

85. Zell R, Delwart E, Gorbalenya AE, Hovi T, King AMQ, Knowles NJ, Lindberg AM, Pallansch MA, Palmenberg AC, Reuter G, Simmonds P, Skern T, Stanway G, Yamashita T. Proposal 2017.008S.A.v1.Orivirus. Create 1 new species (Orivirus $A$ ) in a new genus (Orivirus). http://ictv.global/proposals17/2017.008S.A.v1.Orivirus.zip. Accessed 25 Apr 2018

86. Zell R, Delwart E, Gorbalenya AE, Hovi T, King AMQ, Knowles NJ, Lindberg AM, Pallansch MA, Palmenberg AC, Reuter G, Simmonds P, Skern T, Stanway G, Yamashita T. Proposal 2017.009S.A.v1.Shanbavirus. Create 1 new species (Shanbavirus A) in a new genus (Shanbavirus). http://ictv.global/proposals17/2017.009S.A.v1.Shanbavirus.zip. Accessed 25 Apr 2018

87. Smith DB, Meyers G, Bukh J, Gould EA, Monath T, Muerhoff AS, Pletnev A, Rico-Hesse R, Stapleton JT, Simmonds P and Becher P. Proposal 2017.010S.A.v1.Pestivirus_7sp4spren. Rename four species and create seven species in the genus Pestivirus, family Flaviviridae. http://ictv.global/proposals-17/2017.010S.A.v1.Pesti virus_7sp4spren.zip. Accessed 25 Apr 2018

88. Olendraitė I, Lukhovitskaya NI, Porter SD, Valles SM, Firth AE. Proposal 2017.011S.A.v2.Polycipiviridae. In the order Picornavirales, create family Polycipiviridae comprising three genera, Sopolycivirus, Hupolycivirus and Chipolycivirus. http://ictv.globa 1/proposals-17/2017.011S.A.v2.Polycipiviridae.zip. Accessed 25 Apr 2018 


\section{Affiliations}

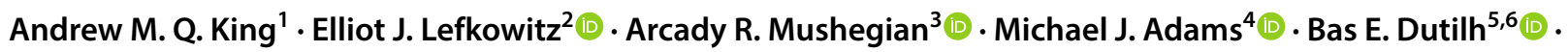

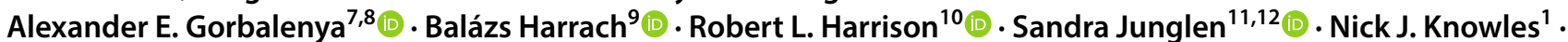

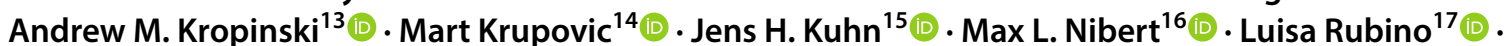

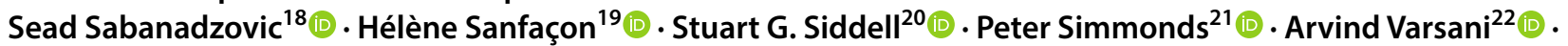
Francisco Murilo Zerbini ${ }^{23}$ (D) Andrew J. Davison ${ }^{24}{ }^{(1)}$

Andrew M. Q. King

amqking@gmail.com

Elliot J. Lefkowitz

elliotl@uab.edu

Michael J. Adams

mike.adams.ictv@gmail.com

Bas E. Dutilh

bedutilh@gmail.com

Alexander E. Gorbalenya

a.e.gorbalenya@lumc.nl

Balázs Harrach

harrach.balazs@agrar.mta.hu

Robert L. Harrison

robert.1.harrison@ars.usda.gov

Sandra Junglen

sandra.junglen@charite.de

Nick J. Knowles

nick.knowles@pirbright.ac.uk

Andrew M. Kropinski

phage.canada@gmail.com

Mart Krupovic

krupovic@pasteur.fr

Jens H. Kuhn

kuhnjens@mail.nih.gov

Max L. Nibert

mnibert@hms.harvard.edu

Luisa Rubino

luisa.rubino@cnr.it

Sead Sabanadzovic

ssabanadzovic@entomology.msstate.edu

Hélène Sanfaçon

helene.sanfacon@agr.gc.ca

Stuart G. Siddell

stuart.siddell@bristol.ac.uk

Peter Simmonds

peter.simmonds@ndm.ox.ac.uk

Arvind Varsani

arvind.varsani@asu.edu

Francisco Murilo Zerbini

zerbini@ufv.br

Andrew J. Davison

andrew.davison@glasgow.ac.uk

1 The Pirbright Institute, Ash Road, Pirbright GU24 0NF, Surrey, UK
2 Department of Microbiology, University of Alabama at Birmingham (UAB), BBRB 276, 845 19th ST South, Birmingham, AL 35294-2170, USA

3 Division of Molecular and Cellular Biosciences, National Science Foundation, 2415 Eisenhower Avenue, Alexandria, VA 22314, USA

4 Minehead, UK

5 Theoretical Biology and Bioinformatics, Department of Biology, Utrecht University, Padualaan 8, $3584 \mathrm{CH}$ Utrecht, The Netherlands

6 Centre for Molecular and Biomolecular Informatics, Radboud University Medical Centre, Geert Grooteplein 28, 6525 GA Nijmegen, The Netherlands

7 Department of Medical Microbiology, Leiden University Medical Center, P. O. Box 9600, E4-P, rm. E4-72, 2300 RC Leiden, The Netherlands

8 Faculty of Bioengineering and Bioinformatics, Lomonosov Moscow State University, Moscow 119899, Russia

9 Centre for Agricultural Research, Hungarian Academy of Sciences, Institute for Veterinary Medical Research, Hungária krt. 21, Budapest 1143, Hungary

10 Invasive Insect Biocontrol and Behavior Laboratory, USDA, 10300 Baltimore Avenue, Bldg 007 Barc-West, Beltsville, MD 20705, USA

11 Institute of Virology, Charité-Universitätsmedizin Berlin, corporate member of Free University Berlin, Humboldt-University Berlin, and Berlin Institute of Health, Rahel-Hirsch-Weg 3, Charitéplatz 1, 10117 Berlin, Germany

12 German Center for Infection Research (DZIF), Berlin, Germany

13 Departments of Food Science and Pathobiology, University of Guelph, Guelph, ON N1G 2W1, Canada

14 Department of Microbiology, Institut Pasteur, 25 rue du Dr Roux, 75015 Paris, France

15 NIH/NIAID/DCR Integrated Research Facility at Fort Detrick (IRF-Frederick), B-8200, Research Plaza, Fort Detrick, Frederick, MD 21702, USA

16 Department of Microbiology and Immunobiology, Harvard Medical School, 77 Ave Louis, Pasteur, Boston, MA 02115, USA

17 Istituto per la Protezione Sostenibile delle Piante, CNR, SS Bari, Via Amendola 165/A, 70126 Bari, Italy

18 Department of Biochemistry, Molecular Biology, Entomology and Plant Pathology, Mississippi State University, 100 Old Hwy 12 Mail Stop 9775, Mississippi State, MS 39762, USA 
19 Summerland Research and Development Centre, Agriculture and Agri-Food Canada, 4200 Highway 97, Summerland, BC VOH 1Z0, Canada

20 Faculty of Biomedical Sciences, School of Cellular and Molecular Medicine, University of Bristol, University Walk, Bristol BS8 1TD, UK

21 Nuffield Department of Experimental Medicine, University of Oxford, Peter Medawar Building, South Parks Road, Oxford OX1 3SY, UK
22 The Biodesign Center for Fundamental and Applied Microbiomics, School of Life Sciences, Center for Evolution and Medicine, Arizona State University, P.O. Box 874701, Tempe, AZ 85287-4701, USA

23 Departamento de Fitopatologia/BIOAGRO, Universidade Federal de Viçosa, Viçosa, MG 36570-900, Brazil

24 MRC-University of Glasgow Centre for Virus Research, Sir Michael Stoker Building, 464 Bearsden Road, Glasgow G61 1QH, UK 